IoAnnis K. Argyros (Lawton, OK)

SAÏD Hilout (Poitiers)

\title{
ON THE CONVERGENCE OF TWO-STEP NEWTON-TYPE METHODS OF HIGH EFFICIENCY INDEX
}

Abstract. We introduce a new idea of recurrent functions to provide a new semilocal convergence analysis for two-step Newton-type methods of high efficiency index. It turns out that our sufficient convergence conditions are weaker, and the error bounds are tighter than in earlier studies in many interesting cases. Applications and numerical examples, involving a nonlinear integral equation of Chandrasekhar type, and a differential equation containing a Green's kernel are also provided.

1. Introduction. In this study we are concerned with the problem of approximating a locally unique solution $x^{\star}$ of the equation

$$
F(x)+G(x)=0,
$$

where $F$ is a Fréchet-differentiable operator defined on a convex subset $\mathcal{D}$ of a Banach space $\mathcal{X}$ with values in a Banach space $\mathcal{Y}$, and $G: \mathcal{D} \rightarrow \mathcal{Y}$ is a continuous operator.

In 1669 Isaac Newton inaugurated his method of solving equations through the use of numerical examples, but did not use the current iterative expression. Later, in 1690, Raphson introduced Newton's method or the so called Newton-Raphson method.

Newton's method is currently and undoubtedly the most popular onepoint iterative procedure for generating a sequence approximating $x^{\star}$. Results on local as well as semilocal convergence of Newton-type methods can be found in [6] and the references there (see also [1]-[5], [7]-[22]).

2010 Mathematics Subject Classification: 65H10, 65G99, 65J15, 47H17, 49M15.

Key words and phrases: two-step Newton-type method, recurrent functions, Banach space, semilocal convergence, efficiency index, Chandrasekhar-type nonlinear equation, differential equation of Hammerstein type. 
One factor that is taken into account, when using one-point iterative methods is the efficiency index: $E P=p^{1 / q}$, where $p$ is the order of convergence of the method, and $q$ is the number of new function values required at each step.

Recently, in the elegant study by Ezquerro and Hernández [13] on Chebyshev's method [6], new third order multipoint iterations are constructed with efficiency index close to Newton's method, and the same region of accessibility.

Motivated by optimization considerations, the study mentioned above, and our works [2]-[5], where modified Newton's method is mixed with Newton's method in order to expand the applicability of the latter, we introduce the two-step Newton-type method (TSNTM):

$$
\begin{aligned}
& x_{0} \in \mathcal{D}, \\
& y_{n}=x_{n}-A_{n}^{-1}\left(F\left(x_{n}\right)+G\left(x_{n}\right)\right), \\
& z_{n}=x_{n}+\alpha\left(y_{n}-x_{n}\right), \\
& x_{n+1}=x_{n}-A_{n}^{-1}\left(\beta\left(F\left(x_{n}\right)+G\left(x_{n}\right)\right)+\gamma\left(F\left(z_{n}\right)+G\left(z_{n}\right)\right)\right) \quad(n \geq 0),
\end{aligned}
$$

where $A_{n}:=A\left(x_{n}\right) \in \mathcal{L}(\mathcal{X}, \mathcal{Y})$, the space of bounded linear operators from $\mathcal{X}$ to $\mathcal{Y}$, and $\alpha, \beta, \gamma$ are numbers chosen so that the sequences $\left\{x_{n}\right\},\left\{y_{n}\right\}$ converge to $x^{\star}$.

Many iterative methods are special cases of (TSTNM). For example, if $\gamma=0, \alpha=\beta=1$, and $A(x)=F^{\prime}(x)(x \in \mathcal{D})$, we obtain Zinčenko's method [22]. Moreover, if $G(x)=0(x \in \mathcal{D})$, we obtain Newton's method, whereas if $A(x)=[x, g(x) ; F]$ ( $g$ is a continuous function and $[x, y ; F]$ is a divided difference of order one), we obtain the secant method in the case $g(x)=x^{+}$, where $x^{+}$is the next iterate. Several other choices are also possible [1]-[22]. In particular, for

$$
\begin{aligned}
& A(x)=F^{\prime}(x), \quad x \in \mathcal{D}, \\
& \alpha \in[0,1], \quad \beta=\frac{\alpha^{2}+\alpha-1}{\alpha^{2}}, \quad \gamma=\frac{1}{\alpha^{2}},
\end{aligned}
$$

we obtain the method introduced in [13], denoted by (TSNM), as a special case of (TSNTM). This method was shown to be of order 3 , with efficiency index $\sqrt[3]{3}$. That is, the efficiency index of this method is between that of Newton's method, $\sqrt{2}$, and secant method, $(1+\sqrt{5}) / 2$. Then it is suggested that we can approximate $F^{\prime}\left(x_{n}\right)$ at each step by a divided difference exactly as we do in Newton's method to obtain the secant method. This way we save one computation, since only the evaluation of a new function is needed at each step. Instead of doing just that, we provide a semilocal convergence analysis for the more general (TSTNM), using our new idea of recurrent function. A favorable comparison between (TSNTM) and Newton-type method (NTM) [13] is given in Remark 2.4. 
The study is organized as follows: the semilocal convergence of (TSNTM) is established in Sections 2 and 3 for $\gamma \neq 0$ and $\gamma=0$, respectively. Numerical examples and special cases are also given in Section 4, involving a differential equation containing a Green's type kernel, and a nonlinear integral equation of Chandrasekhar type appearing in radiative transfer. The proofs of some lemmas are given in the appendix.

2. Semilocal convergence analysis of (TSNTM) for $\gamma \neq 0$. Let $\alpha, \gamma, \mu, K, L, M, N, \eta \geq 0$ and $\ell \in[0,1)$ be given constants. Set

$$
b=\mu+N, \quad c=1+\alpha \gamma .
$$

It is convenient to define scalar sequences $\left\{t_{n}\right\},\left\{s_{n}\right\},\left\{f_{n}\right\},\left\{f_{n}^{1}\right\}$ by

$$
t_{0}=0, \quad s_{0}=\eta,
$$

$$
\begin{gathered}
t_{n+1}=s_{n}+\frac{\alpha \gamma}{1-\ell-L t_{n}}\left(\frac{\alpha K}{2}\left(s_{n}-t_{n}\right)+M t_{n}+b\right)\left(s_{n}-t_{n}\right), \\
t_{1}=s_{0}\left\{1+\alpha \gamma\left(\alpha K s_{0} / 2+b\right)\right\}
\end{gathered}
$$

$$
\begin{aligned}
s_{n+1}= & t_{n+1}+\frac{1}{1-\ell-L t_{n+1}}\left(\frac{K}{2}\left(t_{n+1}-t_{n}\right)^{2}+\left(M t_{n}+b\right)\left(t_{n+1}-t_{n}\right)\right. \\
& \left.+\frac{\alpha^{2} \gamma K}{2}\left(s_{n}-t_{n}\right)^{2}+\alpha \gamma\left(M t_{n}+b\right)\left(s_{n}-t_{n}\right)\right)
\end{aligned}
$$

$$
\begin{aligned}
f_{n}(w)= & \frac{\alpha^{2} \gamma K}{2} w^{n} \eta+\alpha \gamma M\left(1+2 w\left(1+w+\cdots+w^{n-2}\right)+w^{n}\right) \eta \\
& +L w\left(1+2 w\left(1+w+\cdots+w^{n-2}\right)+w^{n}\right) \eta-(1-\ell) w+\alpha \gamma b
\end{aligned}
$$

$$
\begin{aligned}
f_{n}^{1}(w)= & \frac{K}{2}(1+w)^{2} w^{n} \eta+(c+w)\left(M\left(1+2 w\left(1+w+\cdots+w^{n-2}\right) \eta+b\right)\right. \\
& +\frac{\alpha^{2} \gamma K}{2} w^{n} \eta+L M w\left(1+2 w\left(1+w+\cdots+w^{n-1}\right)+w^{n+1}\right) \eta \\
& -(1-\ell) w
\end{aligned}
$$

and functions $f_{\infty}, g, f_{\infty}^{1}$, and $g^{1}$ on $[0,+\infty)$ by

$$
\begin{gathered}
f_{\infty}(w)=(L \eta+1-\ell) w^{2}-(\alpha \gamma b+1-\ell-\alpha \gamma M \eta) w+\alpha \gamma b \\
g(w)=L w^{2}+\left(L+\alpha \gamma M+\alpha^{2} \gamma K / 2\right) w+\alpha \gamma M-\alpha^{2} \gamma K / 2, \\
f_{\infty}^{1}(w)=((\eta+L) M+1-b-\ell) w^{2}+(c M \eta-c b+b+\ell-1) w+c b, \\
g^{1}(w)=(K / 2+L M) w^{3}+(K / 2+M+L M) w^{2} \\
+\left(c+M+\alpha^{2} \gamma K / 2-K / 2\right) w+c M-K / 2-\alpha^{2} \gamma K / 2 .
\end{gathered}
$$


Denote by $w_{2}, w_{\infty}, v, w_{2}^{1}, w_{\infty}^{1}, v^{1}$ the minimal nonnegative zeros of $f_{2}$, $f_{\infty}, g, f_{2}^{1}, f_{\infty}^{1}$, and $g^{1}$, respectively (if they exist).

Set

$$
\begin{gathered}
\delta_{1}=\alpha \gamma(\alpha K / 2+b), \\
\delta_{2}=\frac{1}{\left(1-\ell-L t_{1}\right) \eta}\left(\frac{K}{2} t_{1}^{2}+b t_{1}+\frac{\alpha^{2} \gamma K}{2} \eta^{2}+\alpha \gamma b \eta\right), \quad \eta \neq 0, \\
\delta_{0}=\max \left\{\delta_{1}, \delta_{2}\right\}, \\
w_{0}=\max \left\{w_{\infty}, w_{\infty}^{1}\right\} .
\end{gathered}
$$

The hypotheses of Lemma 2.1 that follows have been left as uncluttered as possible. Note however that the verification of these hypotheses involves only computations at the initial point $x_{0}$. Stronger, but easier to verify conditions can be considered replacing all hypotheses of Lemma 2.1, except (2.15). A set $\left(\mathcal{C}_{0}\right)$ of such conditions is given by:

$$
\begin{gathered}
2 M \leq \alpha K, \quad \alpha \in[0,1], \\
(1-\ell) v-\alpha \gamma b>0, \\
(1-\ell) v^{1}-(c+w) b>0, \\
\eta<\eta_{0}=\min \left\{\eta_{1}, \eta_{2}, \eta_{3}: \eta_{1}>0, f_{2}\left(\eta_{1}\right)=0, \eta_{2}>0, f_{2}^{1}\left(\eta_{2}\right)=0,\right. \\
\left.\eta_{3}>0, L t_{1}+\ell=1\right\} .
\end{gathered}
$$

Indeed, the first condition, and the intermediate value theorem (IVT) applied to the functions $g, g^{1}$ defined on $[0, w]$ for sufficiently large $w>0$, guarantee the existence of zeros $v$ and $v^{1}$, respectively.

The second and third conditions together with (IVT) and the choices of $\eta, \eta_{1}, \eta_{2}$ guarantee the existence of $w_{2}, w_{2}^{1}$ so that (2.16) and (2.17) are satisfied. Moreover, the choice of $\eta$ and $\eta_{3}$ shows that (2.14) is also satisfied. Hence, $(2.15)$ together with the set of conditions $\left(\mathcal{C}_{0}\right)$ can certainly replace the hypotheses of Lemma 2.1.

We can show the following result on majorizing sequences for (TSNTM) (see Appendix).

Lemma 2.1. Assume that there exist minimal nonnegative zeros $w_{2}, w_{\infty}$, $v, w_{2}^{1}, w_{\infty}^{1}, v^{1}$ of functions $f_{2}, f_{\infty}, g, f_{2}^{1}, f_{\infty}^{1}$, and $g^{1}$, respectively, and

$$
\begin{gathered}
L t_{1}+\ell<1, \\
\delta_{0} \leq w_{0} \leq 1, \\
w_{2} \leq v \\
w_{2}^{1} \leq v^{1} .
\end{gathered}
$$

Set

$$
\delta=2 \max \left\{w_{2}, w_{2}^{1}\right\}
$$


Then the scalar sequences $\left\{t_{n}\right\},\left\{s_{n}\right\}(n \geq 0)$ given by (2.2) and (2.3) are increasing, bounded above by

$$
t^{\star \star}=\frac{2+\delta}{2-\delta} \eta,
$$

and converge to a common least upper bound $t^{\star}$ satisfying

$$
0 \leq t^{\star} \leq t^{\star \star} \text {. }
$$

Moreover, the following estimates hold for all $n \geq 0$ :

$$
\begin{aligned}
0 \leq t_{n+1}-s_{n} & \leq \frac{\delta}{2}\left(s_{n}-t_{n}\right) \leq\left(\frac{\delta}{2}\right)^{n+1} \eta, \\
0 \leq s_{n+1}-t_{n+1} & \leq \frac{\delta}{2}\left(s_{n}-t_{n}\right) \leq\left(\frac{\delta}{2}\right)^{n+1} \eta, \\
0 \leq t^{\star}-t_{n} & \leq \frac{2+\delta}{2-\delta}\left(\frac{\delta}{2}\right)^{n} \eta, \\
0 \leq t^{\star}-s_{n} & \leq \frac{3}{2-\delta}\left(\frac{\delta}{2}\right)^{n+1} \eta .
\end{aligned}
$$

We also need a result relating the distances involved in (TSNTM).

Lemma 2.2. If the sequences $\left\{x_{n}\right\},\left\{y_{n}\right\}$ are well defined for all $n \geq 0$, and

$$
(1-\alpha) \gamma=1-\beta \quad(\gamma \neq 0), \quad \text { for some } \beta \geq 0,
$$

then the following hold for all $n \geq 0$ :

$$
\begin{gathered}
x_{n+1}-y_{n}=-\gamma A_{n}^{-1}\left\{\alpha \int_{0}^{1}\left(F^{\prime}\left(x_{n}+\alpha t\left(y_{n}-x_{n}\right)\right)-F^{\prime}\left(x_{n}\right)\right)\left(y_{n}-x_{n}\right) d t\right. \\
\left.+\alpha\left(F^{\prime}\left(x_{n}\right)-A_{n}\right)\left(y_{n}-x_{n}\right)+G\left(z_{n}\right)-G\left(x_{n}\right)\right\}, \\
y_{n+1}-x_{n+1}=-A_{n+1}^{-1} B_{n+1},
\end{gathered}
$$

where

$$
\begin{aligned}
B_{n+1}= & F\left(x_{n+1}\right)+G\left(x_{n+1}\right) \\
= & \int_{0}^{1}\left(F^{\prime}\left(x_{n}+t\left(x_{n+1}-x_{n}\right)\right)-F^{\prime}\left(x_{n}\right)\right)\left(x_{n+1}-x_{n}\right) d t \\
& +\left(F^{\prime}\left(x_{n}\right)-A_{n}\right)\left(x_{n+1}-x_{n}\right)+G\left(x_{n+1}\right)-G\left(x_{n}\right) \\
& -\gamma\left\{\alpha \int_{0}^{1}\left(F^{\prime}\left(x_{n}+\alpha t\left(y_{n}-x_{n}\right)\right)-F^{\prime}\left(x_{n}\right)\right)\left(y_{n}-x_{n}\right) d t\right. \\
& \left.+\alpha\left(F^{\prime}\left(x_{n}\right)-A_{n}\right)\left(y_{n}-x_{n}\right)+G\left(z_{n}\right)-G\left(x_{n}\right)\right\} .
\end{aligned}
$$


Proof. By eliminating $x_{n}$ from the third equation in (TSNTM), we obtain in turn

$$
\begin{aligned}
x_{n+1}-y_{n}= & x_{n}-A_{n}^{-1}\left\{\beta\left(F\left(x_{n}\right)+G\left(x_{n}\right)\right)+\gamma\left(F\left(z_{n}\right)+G\left(z_{n}\right)\right)\right\}-x_{n} \\
& +A_{n}^{-1}\left(F\left(x_{n}\right)+G\left(x_{n}\right)\right) \\
= & -A_{n}^{-1}\left\{(\beta-1)\left(F\left(x_{n}\right)+G\left(x_{n}\right)\right)+\gamma\left(F\left(z_{n}\right)+G\left(z_{n}\right)\right)\right\} \\
= & -\gamma A_{n}^{-1}\left\{\frac{\beta-1}{\gamma}\left(F\left(x_{n}\right)+G\left(x_{n}\right)\right)+\left(F\left(z_{n}\right)+G\left(z_{n}\right)\right)\right\} \\
= & \gamma A_{n}^{-1}\left\{(1-\alpha)\left(F\left(x_{n}\right)+G\left(x_{n}\right)\right)-\left(F\left(z_{n}\right)+G\left(z_{n}\right)\right)\right\}
\end{aligned}
$$

by (2.25). We also have

$$
\begin{aligned}
F\left(z_{n}\right)+G\left(z_{n}\right)= & F\left(z_{n}\right)+G\left(x_{n}\right)+G\left(z_{n}\right)-G\left(x_{n}\right) \\
= & (1-\alpha)\left(F\left(x_{n}\right)+G\left(x_{n}\right)\right)+F\left(z_{n}\right)-F\left(x_{n}\right) \\
& +\alpha\left(F\left(x_{n}\right)+G\left(x_{n}\right)\right)+G\left(z_{n}\right)-G\left(x_{n}\right) \\
= & (1-\alpha)\left(F\left(x_{n}\right)+G\left(x_{n}\right)\right)+F\left(z_{n}\right)-F\left(x_{n}\right)-\alpha A_{n}\left(y_{n}-x_{n}\right) \\
= & (1-\alpha)\left(F\left(x_{n}\right)+G\left(x_{n}\right)\right) \\
& +\alpha \int_{0}^{1}\left(F^{\prime}\left(x_{n}+\alpha t\left(y_{n}-x_{n}\right)\right)-F^{\prime}\left(x_{n}\right)\right)\left(y_{n}-x_{n}\right) d t \\
& +\alpha\left(F^{\prime}\left(x_{n}\right)-A_{n}\right)\left(y_{n}-x_{n}\right)+G\left(z_{n}\right)-G\left(x_{n}\right) .
\end{aligned}
$$

Estimate (2.26) follows from (2.29) and (2.30).

Using (TSNTM), we have

$$
\begin{aligned}
B_{n+1}= & F\left(x_{n+1}\right)+G\left(x_{n+1}\right) \\
= & F\left(x_{n+1}\right)+G\left(x_{n+1}\right)-A_{n}\left(y_{n}-x_{n}\right)-F\left(x_{n}\right)-G\left(x_{n}\right) \\
= & F\left(x_{n+1}\right)-F\left(x_{n}\right)-F^{\prime}\left(x_{n}\right)\left(x_{n+1}-x_{n}\right) \\
& +F^{\prime}\left(x_{n}\right)\left(x_{n+1}-x_{n}\right)-A_{n}\left(y_{n}-x_{n}\right)+G\left(x_{n+1}\right)-G\left(x_{n}\right) \\
= & \int_{0}^{1}\left(F^{\prime}\left(x_{n}+t\left(x_{n+1}-x_{n}\right)\right)-F^{\prime}\left(x_{n}\right)\right)\left(x_{n+1}-x_{n}\right) d t \\
& +\left(F^{\prime}\left(x_{n}\right)-A_{n}\right)\left(x_{n+1}-x_{n}\right)+A_{n}\left(x_{n+1}-x_{n}\right) \\
& \quad-A_{n}\left(y_{n}-x_{n}\right)+G\left(x_{n+1}\right)-G\left(x_{n}\right) \\
& 1 \\
= & \int_{0}^{1}\left(F^{\prime}\left(x_{n}+t\left(x_{n+1}-x_{n}\right)\right)-F^{\prime}\left(x_{n}\right)\right)\left(x_{n+1}-x_{n}\right) d t \\
& +\left(F^{\prime}\left(x_{n}\right)-A_{n}\right)\left(x_{n+1}-x_{n}\right)+A_{n}\left(x_{n+1}-y_{n}\right)+G\left(x_{n+1}\right)-G\left(x_{n}\right) .
\end{aligned}
$$

Estimate (2.27) follows from (2.26) and (2.31).

That completes the proof of Lemma 2.2. 
We shall show the following semilocal convergence theorem for (TSNTM).

TheOREm 2.3. Let $F: \mathcal{D} \subseteq \mathcal{X} \rightarrow \mathcal{Y}$ be a Fréchet-differentiable operator, where $\mathcal{X}, \mathcal{Y}$ are Banach spaces and $\mathcal{D}$ is convex, let $G: \mathcal{D} \rightarrow \mathcal{Y}$ be a continuous operator, and let $A(x) \in \mathcal{L}(\mathcal{X}, \mathcal{Y})$ be an approximation of $F^{\prime}(x)$. Assume that there exist a vector $x_{0} \in \mathcal{D}$, a bounded inverse $A_{0}^{-1}:=A\left(x_{0}\right)^{-1}$ of $A_{0}:=A\left(x_{0}\right)$, and constants $K, L, M, N, \mu, \eta \geq 0, \ell \in[0,1), \alpha, \beta \in[0,1]$, and $\gamma>0$ such that for all $x, y \in \mathcal{D}$ :

$$
\begin{aligned}
& \left\|A_{0}^{-1}\left[F\left(x_{0}\right)+G\left(x_{0}\right)\right]\right\| \leq \eta, \\
& \left\|A_{0}^{-1}\left[F^{\prime}(x)-F^{\prime}(y)\right]\right\| \leq K\|x-y\|, \\
& \left\|A_{0}^{-1}\left[F^{\prime}(x)-A(x)\right]\right\| \leq M\left\|x-x_{0}\right\|+\mu, \\
& \left\|A_{0}^{-1}\left[A(x)-A_{0}\right]\right\| \leq L\left\|x-x_{0}\right\|+\ell, \\
& \left\|A_{0}^{-1}[G(x)-G(y)]\right\| \leq N\|x-y\|, \\
& \bar{U}\left(x_{0}, t^{\star}\right)=\left\{x \in \mathcal{X}:\left\|x-x_{0}\right\| \leq t^{\star}\right\} \subseteq \mathcal{D},
\end{aligned}
$$

and the hypotheses of Lemma 2.1 and (2.25) hold. Then the sequences $\left\{x_{n}\right\}$, $\left\{y_{n}\right\}(n \geq 0)$ generated by $(T S N T M)$ are well defined, remain in $\bar{U}\left(x_{0}, t^{\star}\right)$ for all $n \geq 0$, and converge to a solution $x^{\star} \in \bar{U}\left(x_{0}, t^{\star}\right)$ of the equation $F(x)+G(x)=0$. Moreover, the following estimates hold for all $n \geq 0$ :

$$
\begin{aligned}
\left\|y_{n}-x_{n}\right\| & \leq s_{n}-t_{n}, \\
\left\|x_{n+1}-y_{n}\right\| & \leq t_{n+1}-s_{n}, \\
\left\|x_{n+1}-x_{n}\right\| & \leq t_{n+1}-t_{n}, \\
\left\|y_{n}-x^{\star}\right\| & \leq t^{\star}-s_{n}, \\
\left\|x_{n}-x^{\star}\right\| & \leq t^{\star}-t_{n},
\end{aligned}
$$

where the sequences $\left\{t_{n}\right\},\left\{s_{n}\right\}(n \geq 0)$, and $t^{\star}$ are given in Lemma 2.1. Furthermore, the solution $x^{\star}$ of equation (1.1) is unique in $\bar{U}\left(x_{0}, t^{\star}\right)$ provided that

$$
(K / 2+M+L) t^{\star}+b+\ell<1 .
$$

Proof. We shall show that estimates (2.38)-(2.40) hold for all $n \geq 0$, and $y_{n}, z_{n}, x_{n+1} \in \bar{U}\left(x_{0}, t^{\star}\right)$.

Using (TSNTM), (2.2) for $n=0$, and (2.32), we get

$$
\left\|y_{0}-x_{0}\right\|=\left\|A_{0}^{-1}\left(F\left(x_{0}\right)+G\left(x_{0}\right)\right)\right\| \leq \eta=s_{0}-t_{0},
$$

which implies $y_{0} \in \bar{U}\left(x_{0}, t^{\star}\right)$, and (2.38) holds for $n=0$ by the definition of $t^{\star}$.

We also have

$$
\begin{aligned}
z_{0}-x_{0}=\alpha\left(y_{0}-x_{0}\right) & \Rightarrow\left\|z_{0}-x_{0}\right\|=\alpha\left\|y_{0}-x_{0}\right\| \leq \alpha \eta \leq \eta \\
& \Rightarrow z_{0} \in \bar{U}\left(x_{0}, t^{\star}\right) .
\end{aligned}
$$

Hence, $x_{1}$ is well defined. 
Using (2.1), (2.2), (2.26), (2.33), (2.34), (2.44), and (2.45) we obtain

$$
\begin{aligned}
\left\|x_{1}-y_{1}\right\| \leq & \gamma\left(\frac{\alpha^{2} K}{2}\left\|y_{0}-x_{0}\right\|^{2}+\alpha\left(M\left\|x_{0}-x_{0}\right\|+\mu\right)\left\|y_{0}-x_{0}\right\|\right. \\
& \left.+N\left\|z_{0}-x_{0}\right\|\right) \\
\leq & \alpha \gamma\left(\frac{\alpha K}{2}\left(s_{0}-t_{0}\right)+b\right)\left(s_{0}-t_{0}\right) \leq t_{1}-s_{0},
\end{aligned}
$$

which shows (2.39) for $n=0$.

We also have

$$
\begin{aligned}
\left\|x_{1}-x_{0}\right\| & =\left\|\left(x_{1}-y_{0}\right)+\left(y_{0}-x_{0}\right)\right\| \\
& \leq\left\|x_{1}-y_{0}\right\|+\left\|y_{0}-x_{0}\right\| \\
& \leq t_{1}-s_{0}+s_{0}-t_{0}=t_{1}-t_{0} \leq t^{\star},
\end{aligned}
$$

which implies (2.40) holds for $n=0$, and $x_{1} \in \bar{U}\left(x_{0}, t^{\star}\right)$.

Let us assume that (2.38)-(2.40) and $y_{k}, z_{k}, x_{k+1} \in \bar{U}\left(x_{0}, t^{\star}\right)$ hold for all $k \leq n-1$. Let $u \in \bar{U}\left(x_{0}, t^{\star}\right)$. Then, using (A.4) and (2.35), we get

$$
\left\|A_{0}^{-1}\left[A(u)-A_{0}\right]\right\| \leq L\left\|u-x_{0}\right\|+\ell \leq L t^{\star}+\ell<1 .
$$

It follows from (2.48) and the Banach lemma on invertible operators [6], [15] that $A(u)^{-1}$ exists, with

$$
\left\|A(u)^{-1} A_{0}\right\| \leq\left(1-\ell-L\left\|u-x_{0}\right\|\right)^{-1} .
$$

In particular, for $u=x_{k}$, we have

$$
\left\|x_{k}-x_{0}\right\| \leq \sum_{i=1}^{k}\left\|x_{i}-x_{i-1}\right\| \leq \sum_{i=1}^{k}\left(t_{i}-t_{i-1}\right)=t_{k}-t_{0} \leq t^{\star},
$$

and, similarly for $u=x_{k+1}$,

$$
\left\|x_{k+1}-x_{0}\right\| \leq t_{k+1}-t_{0} \leq t^{\star},
$$

Hence, using (2.49), we have

$$
\begin{aligned}
\left\|A_{k}^{-1} A_{0}\right\| & \leq\left(1-\ell-L t_{k}\right)^{-1} \\
\left\|A_{k+1}^{-1} A_{0}\right\| & \leq\left(1-\ell-L t_{k+1}\right)^{-1} .
\end{aligned}
$$

Using (2.2), (2.26), (2.33), (2.34), (2.36), (2.50), (2.52), and the induction hypotheses, we get in turn

$$
\begin{aligned}
\left\|x_{k+1}-y_{k}\right\| \leq & \gamma\left\|A_{k}^{-1} A_{0}\right\|\left(\frac{\alpha^{2} K}{2}\left\|y_{k}-x_{k}\right\|^{2}\right. \\
& \left.+\alpha\left(M\left\|x_{k}-x_{0}\right\|+\mu\right)\left\|y_{k}-x_{k}\right\|+N\left\|z_{k}-x_{k}\right\|\right)
\end{aligned}
$$




$$
\begin{aligned}
& \leq \frac{\alpha \gamma}{1-\ell-L t_{k}}\left(\frac{\alpha K}{2}\left\|y_{k}-x_{k}\right\|+M\left\|x_{k}-x_{0}\right\|+b\right)\left\|y_{k}-x_{k}\right\| \\
& \leq \frac{\alpha \gamma}{1-\ell-L t_{k}}\left(\frac{\alpha K}{2}\left(s_{k}-t_{k}\right)+M t_{k}+b\right)\left(s_{k}-t_{k}\right)=t_{k+1}-s_{k},
\end{aligned}
$$

which shows (2.39) for all $n \geq 0$.

Moreover, using (2.3), (2.27), (2.33), (2.34), (2.36), (2.53), and the induction hypotheses, we obtain, as in (2.54),

$$
\begin{aligned}
\left\|y_{k+1}-x_{k+1}\right\| \leq & \left\|A_{k+1}^{-1} A_{0}\right\|\left\|A_{0}^{-1}\left(F\left(x_{k+1}\right)+G\left(x_{k+1}\right)\right)\right\| \\
\leq & \frac{1}{1-\ell-L t_{k+1}}\left(\frac{K}{2}\left(t_{k+1}-t_{k}\right)^{2}+M\left(t_{k}+\mu\right)\left(t_{k+1}-t_{k}\right)\right. \\
& +N\left(t_{k+1}-t_{k}\right)+\frac{\alpha^{2} \gamma K}{2}\left(s_{k}-t_{k}\right)^{2}+\alpha \gamma\left(M t_{k}+\mu\right)\left(s_{k}-t_{k}\right) \\
& \left.+N \alpha \gamma\left(s_{k}-t_{k}\right)\right) \\
= & s_{k+1}-t_{k+1},
\end{aligned}
$$

which shows (2.38) for all $n \geq 0$.

We also have

$$
\begin{aligned}
\left\|x_{k+1}-x_{k}\right\| & \leq\left\|x_{k+1}-y_{k}\right\|+\left\|y_{k}-x_{k}\right\| \leq\left(t_{k+1}-s_{k}\right)+\left(s_{k}-t_{k}\right) \\
& =t_{k+1}-t_{k}
\end{aligned}
$$

which shows (2.40) for all $n \geq 0$.

Furthermore, we have

$$
\begin{aligned}
\left\|y_{k+1}-x_{0}\right\| & \leq\left\|y_{k+1}-x_{k+1}\right\|+\left\|x_{k+1}-x_{0}\right\| \\
& \leq\left(s_{k+1}-t_{k+1}\right)+\left(t_{k+1}-t_{0}\right)=s_{k+1}-t_{0} \leq t^{\star}, \\
\left\|z_{k+1}-x_{0}\right\| & =\left\|(1-\alpha)\left(x_{k+1}-x_{0}\right)+\alpha\left(y_{k+1}-x_{0}\right)\right\| \\
& \leq(1-\alpha)\left\|x_{k+1}-x_{0}\right\|+\alpha\left\|y_{k+1}-x_{0}\right\| \\
& \leq(1-\alpha) t_{k+1}+\alpha s_{k+1}=t_{k+1}+\alpha\left(s_{k+1}-t_{k+1}\right) \\
& \leq t_{k+1}+s_{k+1}-t_{k+1}=s_{k+1} \leq t^{\star}
\end{aligned}
$$

which implies $y_{n}, z_{n} \in \bar{U}\left(x_{0}, t^{\star}\right)$ for all $n \geq 0$, and

$$
\left\|z_{k+1}-x_{k+1}\right\| \leq \alpha\left\|y_{k+1}-x_{k+1}\right\| \leq \alpha\left(s_{k+1}-t_{k+1}\right) .
$$

That completes the induction.

Lemma 2.1 implies that the sequences $\left\{t_{n}\right\},\left\{s_{n}\right\}$ are Cauchy. Hence, $\left\{x_{n}\right\},\left\{y_{n}\right\}(n \geq 0)$ are also Cauchy sequences in the Banach space $\mathcal{X}$, and as such they converge to a common limit $x^{\star} \in \bar{U}\left(x_{0}, t^{\star}\right)\left(\right.$ since $\bar{U}\left(x_{0}, t^{\star}\right)$ is a closed set). 
By letting $k \rightarrow \infty$ in (2.55), we obtain $F\left(x^{\star}\right)+G\left(x^{\star}\right)=0$. Estimates (2.41) and (2.42) follow from (2.38) and (2.39) by using standard majorization techniques [1], [6], [15].

Finally, to show uniqueness, let $y^{\star} \in \bar{U}\left(x_{0}, t^{\star}\right)$ with $F\left(y^{\star}\right)+G\left(y^{\star}\right)=0$. Then, using (TSNTM), (2.1), (2.33), (2.34), (2.36), (2.43), (2.52), and the identity

$$
\begin{aligned}
y^{\star}-x_{k+1}= & A_{k}^{-1} A_{0}\left\{A _ { 0 } ^ { - 1 } \left(\int_{0}^{1}\left(F^{\prime}\left(x_{k}+\theta\left(y^{\star}-x_{k}\right)\right)-F^{\prime}\left(x_{k}\right)\right) d \theta\right.\right. \\
& \left.\left.+\left(F^{\prime}\left(x_{k}\right)-A_{k}\right)\right)\left(y^{\star}-x_{k}\right)+A_{0}^{-1}\left(G\left(y^{\star}\right)-G\left(x_{k}\right)\right)\right\},
\end{aligned}
$$

we obtain

$$
\begin{aligned}
\left\|y^{\star}-y_{k}\right\| \leq & \left(1-\ell-L t_{k}\right)^{-1}\left\{\left(\int_{0}^{1}\left\|A_{0}^{-1}\left(F^{\prime}\left(x_{k}+\theta\left(y^{\star}-x_{k}\right)\right)-F^{\prime}\left(x_{k}\right)\right)\right\| d \theta\right.\right. \\
& \left.\left.+\left\|A_{0}^{-1}\left(F^{\prime}\left(x_{k}\right)-A_{k}\right)\right\|\right)\left\|y^{\star}-x_{k}\right\|+\left\|A_{0}^{-1}\left(G\left(x_{k}\right)-G\left(y^{\star}\right)\right)\right\|\right\} \\
\leq & \left(1-\ell-L t^{\star}\right)^{-1}\left(\frac{K}{2}\left\|y^{\star}-x_{k}\right\|+M\left\|x_{k}-x_{0}\right\|+b\right)\left\|y^{\star}-x_{k}\right\| \\
\leq & \left(1-\ell-L t^{\star}\right)^{-1}\left(\frac{K}{2} t^{\star}+M t^{\star}+b\right)\left\|y^{\star}-x_{k}\right\| \\
< & \left\|y^{\star}-x_{k}\right\| \quad(\text { by }(2.43)),
\end{aligned}
$$

which implies $\lim _{k \rightarrow \infty} x_{k}=y^{\star}$. But we have shown $\lim _{k \rightarrow \infty} x_{k}=x^{\star}$. Hence, we deduce $x^{\star}=y^{\star}$.

That completes the proof of Theorem 2.3.

Remark 2.4. (a) Note that $t^{\star}$ can be replaced by $t^{\star \star}$ given by $(2.19)$ in all hypotheses of Theorem 2.3.

(b) To compare our results with the corresponding ones in [13] for $A(x)=$ $F^{\prime}(x), G(x)=0(x \in \mathcal{D})$, and $\alpha, \beta, \gamma$ given by (1.2), let us define majorizing sequences $\left\{\bar{t}_{n}\right\},\left\{\bar{s}_{n}\right\}$ essentially used in [13]:

$$
\begin{aligned}
& \bar{t}_{0}=0, \quad \bar{s}_{0}=\eta \\
& \bar{t}_{n+1}=\bar{s}_{n}+\frac{K\left(\bar{s}_{n}-\bar{t}_{n}\right)^{2}}{2\left(1-K \bar{t}_{n}\right)} \quad(n \geq 0) \\
& \bar{s}_{n}=\bar{t}_{n}+\frac{K\left(\left(\bar{s}_{n-1}-\bar{t}_{n-1}\right)^{2}+\left(\bar{t}_{n}-\bar{t}_{n-1}\right)^{2}\right)}{2\left(1-K \bar{t}_{n}\right)} \quad(n \geq 1) .
\end{aligned}
$$

A sufficient convergence condition given in affine invariant form is

$$
h=K \eta<.3266 \text {. }
$$


(c) In view of the proof of Theorem 2.3, (2.2), (2.3), we note that the scalar sequences $\left\{t_{n}\right\},\left\{s_{n}\right\}$ given by

$$
\begin{aligned}
& t_{0}=0, \quad s_{0}=\eta, \quad t_{1}=s_{0}+\frac{L}{2}\left(s_{0}-t_{0}\right)^{2}, \\
& t_{n+1}=s_{n}+\frac{K\left(s_{n}-t_{n}\right)^{2}}{2\left(1-L t_{n}\right)} \quad(n \geq 1), \\
& s_{1}=t_{1}+\frac{K\left(\left(s_{0}-t_{0}\right)^{2}+\left(t_{1}-t_{0}\right)^{2}\right)}{2\left(1-L t_{1}\right)}, \\
& s_{n}=t_{n}+\frac{K\left(\left(s_{n-1}-t_{n-1}\right)^{2}+\left(t_{n}-t_{n-1}\right)^{2}\right)}{2\left(1-L t_{n}\right)} \quad(n \geq 2),
\end{aligned}
$$

are also majorizing sequences for $\left\{x_{n}\right\},\left\{y_{n}\right\}$.

Note that in general

$$
L \leq K,
$$

and $K / L$ can be large (see Section 4 for examples).

An inductive argument for $L<K$ shows

$$
\begin{array}{rlrl}
t_{n} & \leq \bar{t}_{n} & & (n \geq 1), \\
s_{n} & \leq \bar{s}_{n} & & (n \geq 1), \\
t_{n+1}-s_{n} & \leq \bar{t}_{n+1}-\bar{s}_{n} & & (n \geq 0), \\
s_{n+1}-t_{n+1} & \leq \bar{s}_{n+1}-\bar{t}_{n+1} & & (n \geq 0), \\
t^{\star} \leq \overline{t^{\star}} & =\lim _{n \rightarrow \infty} \bar{t}_{n}=\lim _{n \rightarrow \infty} \bar{s}_{n} .
\end{array}
$$

Hence, under condition (2.63), the sequences $\left\{t_{n}\right\},\left\{s_{n}\right\}$ are tighter than $\left\{\bar{t}_{n}\right\},\left\{\bar{s}_{n}\right\}$, and are also majorizing for $\left\{x_{n}\right\},\left\{y_{n}\right\}$. Moreover, the information on the location of the solution is at least as precise as in [13]. Note also that a direct comparison between our results and the ones in [13] cannot be done, since our sufficient convergence conditions (see Lemma 2.1) differ from (2.63). However, since the information $L<K$ is not used in [13], and in view of (2.66)-(2.70), one expects to be able to find cases (see e.g. Section 4) where (2.63) is violated but the hypotheses of Lemma 2.1 hold. Note also that the hypotheses of Lemma 2.1 involve only computations at the initial guess $x_{0}$.

3. Semilocal convergence analysis of (TSNTM) for $\gamma=0$. In this case, it only makes sense to set $\alpha=\beta=1$ in (TSNTM). Hence, (TSNTM) becomes (NTM):

$$
x_{n+1}=x_{n}-A_{n}^{-1}\left(F\left(x_{n}\right)+G\left(x_{n}\right)\right) \quad\left(x_{0} \in \mathcal{D}, n \geq 0\right) .
$$


Lemma 3.1. Assume that there exist constants $K, M, \eta>0$ and $\mu, \ell \geq 0$ such that

$$
\begin{aligned}
2 M & <K, \\
(K+2 L) \eta & <2(1-\ell-\mu),
\end{aligned}
$$

and the quadratic polynomial $f_{1}$ given by

$$
f_{1}(s)=2 L \eta s^{2}-(2(1-\ell-L \eta)-K \eta) s+2(M \eta+\mu)
$$

has a minimal root in $(0,1)$, denoted by $s_{1}$. Moreover, assume that for

$$
\begin{gathered}
\delta_{0}=\frac{K \eta+2 \mu}{1-L \eta-\ell}, \\
\delta_{+}=\frac{2(K-2 M)}{K+\sqrt{K^{2}-8 L(2 M-K)}}, \quad \delta_{\infty}=2 s_{\infty},
\end{gathered}
$$

where $s_{\infty}$ is the minimal root in $(0,1)$ of the equation

$$
\bar{f}_{\infty}(s)=(1-\ell) s^{2}-(1-\ell-L \eta+\mu) s+M \eta+\mu=0
$$

we have

$$
\begin{aligned}
& \delta_{0} \leq \delta_{\infty}, \\
& s_{1} \leq \delta_{+} .
\end{aligned}
$$

Set

$$
\delta=2 s_{1} .
$$

Then the scalar sequence $\left\{t_{n}\right\}(n \geq 0)$ given by

$$
\begin{aligned}
t_{0} & =0, \quad t_{1}=\eta, \\
t_{n+2} & =t_{n+1}+\frac{K\left(t_{n+1}-t_{n}\right)+2\left(M t_{n}+\mu\right)}{2\left(1-L t_{n+1}-\ell\right)}\left(t_{n+1}-t_{n}\right)
\end{aligned}
$$

is increasing, bounded above by

$$
t^{\star \star}=\frac{2 \eta}{2-\delta},
$$

and converges to its least upper bound $t^{\star} \in\left[0, t^{\star \star}\right]$. Moreover, the following estimates hold for all $n \geq 1$ :

$$
t_{n+1}-t_{n} \leq \frac{\delta}{2}\left(t_{n}-t_{n-1}\right) \leq\left(\frac{\delta}{2}\right)^{n} \eta
$$

and

$$
t^{\star}-t_{n} \leq \frac{2 \eta}{2-\delta}\left(\frac{\delta}{2}\right)^{n} .
$$

REMARK 3.2. Note that by applying the intermediate value theorem to $f_{1}$ for $s \in[0,1]$, we see that (3.3) and the condition on the existence of $s_{1}$ 
can be replaced by the condition

$$
(K+4 L+2 M) \eta<2(1-\ell-\mu) .
$$

Another set of replacement conditions is given by $\Delta \geq 0$ and

$$
\max \{(4 L+2 M+K) \eta+2 \mu,(6 L+K) \eta\}<2(1-\ell),
$$

where $\Delta$ is the discriminant of $f_{1}$.

We shall provide a semilocal convergence analysis for (NTM).

TheOREm 3.3. Let $F: \mathcal{D} \subseteq \mathcal{X} \rightarrow \mathcal{Y}$ be a Fréchet-differentiable operator, where $\mathcal{D}$ is an open convex subset of $\mathcal{X}$, let $G: \mathcal{D} \rightarrow \mathcal{Y}$ be a continuous operator, and let $A(x) \in \mathcal{L}(\mathcal{X}, \mathcal{Y})$ be an approximation of $F^{\prime}(x)$. Assume that there exist $x_{0} \in \mathcal{D}$, a bounded inverse $A_{0}^{-1}$ of $A_{0}=A\left(x_{0}\right)$, and constants $K, L, M, \eta>0$ and $\mu_{0}, \mu_{1}, \ell \geq 0$ such that for all $x, y \in \mathcal{D}$ :

$$
\begin{aligned}
& \left\|A_{0}^{-1}\left(F\left(x_{0}\right)+G\left(x_{0}\right)\right)\right\| \leq \eta, \\
& \left\|A_{0}^{-1}\left(F^{\prime}(x)-F^{\prime}(y)\right)\right\| \leq K\|x-y\|, \\
& \left\|A_{0}^{-1}\left(F^{\prime}(x)-A(x)\right)\right\| \leq M\left\|x-x_{0}\right\|+\mu_{0}, \\
& \left\|A_{0}^{-1}\left(A(x)-A_{0}\right)\right\| \leq L\left\|x-x_{0}\right\|+\ell, \\
& \left\|A_{0}^{-1}(G(x)-G(y))\right\| \leq \mu_{1}\|x-y\|, \\
& \bar{U}\left(x_{0}, t^{\star}\right)=\left\{x \in \mathcal{X},\left\|x-x_{0}\right\| \leq t^{\star}\right\} \subseteq \mathcal{D},
\end{aligned}
$$

and the hypotheses of Lemma 3.1 hold with $\mu=\mu_{0}+\mu_{1}$. Then the sequence $\left\{x_{n}\right\}(n \geq 0)$ generated by $(N T M)$ is well defined, remains in $\bar{U}\left(x_{0}, t^{\star}\right)$ for all $n \geq 0$, and converges to a solution $x^{\star}$ of the equation $F(x)+G(x)=0$ in $\bar{U}\left(x_{0}, t^{\star}\right)$. Moreover, the following estimates hold for all $n \geq 0$ :

$$
\begin{aligned}
\left\|x_{n+1}-x_{n}\right\| & \leq t_{n+1}-t_{n}, \\
\left\|x_{n}-x^{\star}\right\| & \leq t^{\star}-t_{n},
\end{aligned}
$$

where the sequence $\left\{t_{n}\right\}(n \geq 0)$ and $t^{\star}$ are given in Lemma 3.1. Furthermore, the solution $x^{\star}$ of equation (1.1) is unique in $\bar{U}\left(x_{0}, t^{\star}\right)$ provided that

$$
(K / 2+M+L) t^{\star}+\mu+\ell<1 .
$$

Proof. We shall show by induction on $m \geq 0$ that

$$
\begin{gathered}
\left\|x_{m+1}-x_{m}\right\| \leq t_{m+1}-t_{m}, \\
\bar{U}\left(x_{m+1}, t^{\star}-t_{m+1}\right) \subseteq \bar{U}\left(x_{m}, t^{\star}-t_{m}\right) .
\end{gathered}
$$

For every $z \in \bar{U}\left(x_{1}, t^{\star}-t_{1}\right)$,

$$
\left\|z-x_{0}\right\| \leq\left\|z-x_{1}\right\|+\left\|x_{1}-x_{0}\right\| \leq t^{\star}-t_{1}+t_{1}=t^{\star}-t_{0}
$$

implies $z \in \bar{U}\left(x_{0}, t^{\star}-t_{0}\right)$. We also have

$$
\left\|x_{1}-x_{0}\right\|=\left\|A_{0}^{-1}\left[F\left(x_{0}\right)+G\left(x_{0}\right)\right]\right\| \leq \eta=t_{1}-t_{0} .
$$


That is, (3.20) and (3.21) hold for $m=0$. Given they hold for $n \leq m$, then

$$
\left\|x_{m+1}-x_{0}\right\| \leq \sum_{i=1}^{m+1}\left\|x_{i}-x_{i-1}\right\| \leq \sum_{i=1}^{m+1}\left(t_{i}-t_{i-1}\right)=t_{m+1}-t_{0}=t_{m+1},
$$

and

$$
\left\|x_{m}+\theta\left(x_{m+1}-x_{m}\right)-x_{0}\right\| \leq t_{m}+\theta\left(t_{m+1}-t_{m}\right) \leq t^{\star},
$$

for all $\theta \in(0,1)$.

Using (3.3), (3.16), and the induction hypotheses, we get

$$
\begin{aligned}
\left\|A_{0}^{-1}\left[A_{m+1}-A_{0}\right]\right\| & \leq L\left\|x_{m+1}-x_{0}\right\|+\ell \leq L\left(t_{m+1}-t_{0}\right)+\ell \\
& \leq L t_{m+1}+\ell<1
\end{aligned}
$$

by (A.26).

It follows from (3.22) and the Banach lemma on invertible operators [6], [15] that $A_{m+1}^{-1}$ exists, and

$$
\left\|A_{m+1}^{-1} A_{0}\right\| \leq\left(1-\ell-L t_{m+1}\right)^{-1} .
$$

Using (3.1), we obtain the approximation

$$
\begin{aligned}
= & -A_{m+1}^{-1} A_{0} A_{0}^{-1}\left(\int_{0}^{1}\left[F^{\prime}\left(x_{m+1}+\theta\left(x_{m}-x_{m+1}\right)\right)-F^{\prime}\left(x_{m}\right)\right]\left(x_{m+1}-x_{m}\right) d \theta\right. \\
& \left.+\left(F^{\prime}\left(x_{m}\right)-A_{m}\right)\left(x_{m+1}-x_{m}\right)+G\left(x_{m+1}\right)-G\left(x_{m}\right)\right)
\end{aligned}
$$

Using (3.14), (3.15), (3.17), (3.23), (3.24), and the induction hypothesis, we obtain in turn

$$
\begin{aligned}
&\left\|x_{m+2}-x_{m+1}\right\| \\
& \leq\left(1-\ell-L t_{m+1}\right)^{-1}\left(\frac{K}{2}\left\|x_{m+1}-x_{m}\right\|^{2}\right. \\
&\left.\quad+\left(M\left\|x_{m}-x_{0}\right\|+\mu_{0}\right)\left\|x_{m+1}-x_{m}\right\|+\mu_{1}\left\|x_{m+1}-x_{m}\right\|\right) \\
& \leq\left(1-\ell-L t_{m+1}\right)^{-1}\left(\frac{K}{2}\left(t_{m+1}-t_{m}\right)+M t_{m}+\mu\right)\left(t_{m+1}-t_{m}\right) \\
&= t_{m+2}-t_{m+1},
\end{aligned}
$$

which shows (3.20) for all $m \geq 0$.

Thus, for every $z \in \bar{U}\left(x_{m+2}, t^{\star}-t_{m+2}\right)$, we have

$$
\begin{aligned}
\left\|z-x_{m+1}\right\| & \leq\left\|z-x_{m+2}\right\|+\left\|x_{m+2}-x_{m+1}\right\| \\
& \leq t^{\star}-t_{m+2}+t_{m+2}-t_{m+1}=t^{\star}-t_{m+1},
\end{aligned}
$$

which shows (3.21) for all $m \geq 0$. 
Lemma 3.1 implies that the sequence $\left\{t_{n}\right\}$ is Cauchy. Moreover, it follows from (3.20) and (3.21) that $\left\{x_{n}\right\}(n \geq 0)$ is also a Cauchy sequence in the Banach space $\mathcal{X}$, and as such it converges to some $x^{\star} \in \bar{U}\left(x_{0}, t^{\star}\right)$.

By letting $m \rightarrow \infty$ in (3.25), we obtain $F\left(x^{\star}\right)+G\left(x^{\star}\right)=0$. Furthermore estimate (3.19) is obtained from (3.18) by using standard majorization techniques [1], [6], [15]. Finally, to show that $x^{\star}$ is the unique solution of (1.1) in $\bar{U}\left(x_{0}, t^{\star}\right)$, as in (3.24) and (3.25), we get in turn for $y^{\star} \in \bar{U}\left(x_{0}, t^{\star}\right)$, with $F\left(y^{\star}\right)+G\left(y^{\star}\right)=0$, the estimate

$$
\begin{aligned}
& \left\|y^{\star}-x_{m+1}\right\| \\
\leq & \left\|A_{m}^{-1} A_{0}\right\|\left\{\left(\int_{0}^{1}\left\|A_{0}^{-1}\left(F^{\prime}\left(x_{m}+\theta\left(y^{\star}-x_{m}\right)\right)-F^{\prime}\left(x_{m}\right)\right)\right\| d \theta\right.\right. \\
& \left.\left.+\left\|A_{0}^{-1}\left[F^{\prime}\left(x_{m}\right)-A_{m}\right]\right\|\right)\left\|y^{\star}-x_{m}\right\|+\left\|A_{0}^{-1}\left[G\left(x_{m}\right)-G\left(y^{\star}\right)\right]\right\|\right\} \\
\leq & \left(1-L t_{m+1}\right)^{-1}\left(\frac{K}{2}\left\|y^{\star}-x_{m}\right\|^{2}+\left(M\left\|x_{m}-x_{0}\right\|+\mu\right)\left\|y^{\star}-x_{m}\right\|\right) \\
\leq & \left(1-L t_{m+1}\right)^{-1}\left(\frac{K}{2}\left(t^{\star}-t_{m}\right)+M t_{m}+\mu\right)\left\|y^{\star}-x_{m}\right\| \\
\leq & \left(1-L t^{\star}\right)^{-1}\left(\frac{K}{2}\left(t^{\star}-t_{0}\right)+M t^{\star}+\mu\right)\left\|x^{\star}-x_{m}\right\|<\left\|y^{\star}-x_{m}\right\|,
\end{aligned}
$$

by the uniqueness hypothesis.

It follows by (3.26) that $\lim _{m \rightarrow \infty} x_{m}=y^{\star}$. But we have shown $\lim _{m \rightarrow \infty} x_{m}$ $=x^{\star}$. Hence, we deduce $x^{\star}=y^{\star}$.

That completes the proof of Theorem 3.3.

\section{Special cases and applications}

Application $4.1(\gamma=0)$. Using (3.13)-(3.16) and the hypothesis

$$
h_{K}=\sigma \eta \leq \frac{1}{2}(1-b)^{2}, \quad \mu+\ell<1,
$$

where $\sigma=\max \{K, M+L\}$ with $b=\mu+\ell$, a semilocal convergence theorem was provided in [9]-[12], [16]-[22].

(a) Let us compare the error bounds in this case. The majorizing sequence given in [9]-[12], [16]-[22], is

$$
\begin{aligned}
& v_{0}=0, \quad v_{1}=\eta, \\
& v_{n+2}=v_{n+1}+\frac{f\left(v_{n+1}\right)}{q\left(v_{n+1}\right)} \quad(n \geq 0),
\end{aligned}
$$

where

$$
f(v)=\frac{\sigma}{2} v^{2}-(1-b) v+\eta, \quad q(v)=1-L v-\ell .
$$


We now show that the error bounds obtained in Theorem 3.3 are more precise than the corresponding ones in the above references using (4.1).

Proposition 4.2. Under the hypotheses of Theorem 3.3, and condition (4.1), the following error bounds hold:

$$
\begin{aligned}
t_{n+1} & \leq v_{n+1} & & (n \geq 1), \\
t_{n+1}-t_{n} & \leq v_{n+1}-v_{n} & & (n \geq 1), \\
t^{\star}-t_{n} & \leq v^{\star}-v_{n} & & (n \geq 0), \\
t^{\star} & \leq v^{\star} . & &
\end{aligned}
$$

Moreover, strict inequality holds in (4.3) and (4.4) if $K<M+L$.

Proof. We use induction on $m$ to show (4.3) and (4.4). For $n=0$ in (2.19) we obtain

$$
\begin{aligned}
t_{2}-\eta & =\frac{\frac{K}{2} \eta^{2}+\mu \eta}{1-\ell-L \eta} \leq \frac{\frac{\sigma}{2} \eta^{2}+(M \cdot 0+\mu) \eta}{1-\ell-L \eta} \\
& \leq \frac{\frac{\sigma}{2} \eta^{2}+M(\eta-0)+\mu(\eta-0)-q(0)(\eta-0)+f(0)}{q(\eta)} \\
& \leq \frac{\frac{\sigma}{2} v_{1}^{2}-(1-\mu-\ell) v_{1}+\eta-(\sigma-M-L) v_{0}\left(v_{1}-v_{0}\right)}{q\left(v_{1}\right)} \\
& \leq \frac{f\left(v_{1}\right)}{q\left(v_{1}\right)}=v_{2}-v_{1},
\end{aligned}
$$

and $t_{2} \leq v_{2}$.

Assume that

$$
t_{i+1} \leq v_{i+1}, \quad t_{i+1}-t_{i} \leq v_{i+1}-v_{i} .
$$

Using (2.19), (4.2), and (4.7), we obtain in turn

$$
\begin{aligned}
& t_{i+2}-t_{i+1} \\
& =\frac{\frac{K}{2}\left(t_{i+1}-t_{i}\right)^{2}+\left(M t_{i}+\mu\right)\left(t_{i+1}-t_{i}\right)}{1-\ell-L t_{i+1}} \\
& \leq \frac{\frac{\sigma}{2}\left(v_{i+1}-v_{i}\right)^{2}+\left(M v_{i}+\mu\right)\left(v_{i+1}-v_{i}\right)}{q\left(v_{i+1}\right)} \\
& =\frac{\frac{\sigma}{2}\left(v_{i+1}-v_{i}\right)^{2}+M\left(v_{i+1}-v_{i}\right) v_{i}+\mu\left(v_{i+1}-v_{i}\right)-q\left(v_{i}\right)\left(v_{i+1}-v_{i}\right)+f\left(v_{i}\right)}{q\left(v_{i+1}\right)} \\
& =\frac{\frac{\sigma}{2} v_{i+1}^{2}-(1-\mu-\ell) v_{i+1}+\eta-(\sigma-M-L) v_{i}\left(v_{i+1}-v_{i}\right)}{q\left(v_{i+1}\right)} \\
& \leq \frac{f\left(v_{i+1}\right)}{q\left(v_{i+1}\right)}=v_{i+2}-v_{i+1},
\end{aligned}
$$

which shows (4.3) and (4.4) for all $n \geq 1$. 
For $j \geq 0$, we get

$$
\begin{aligned}
t_{i+j}-t_{i} & \leq\left(t_{i+j}-t_{i+j-1}\right)+\left(t_{i+j-1}-t_{i+j-2}\right)+\cdots+\left(t_{i+1}-t_{i}\right) \\
& \leq\left(v_{i+j}-v_{i+j-1}\right)+\left(v_{i+j-1}-v_{i+j-2}\right)+\cdots+\left(v_{i+1}-v_{i}\right) \\
& \leq v_{i+1}-v_{i} .
\end{aligned}
$$

By letting $j \rightarrow \infty$ in (4.8) we obtain (4.5).

Finally, (4.5) implies (4.6) (since $\left.t_{1}=v_{1}=0\right)$ ). It can easily be seen from (2.19) and (4.2) that strict inequality holds in (4.3) and (4.4) if $K<M+L$.

That completes the proof of Proposition 4.2.

Note also that the above advantages hold even if the hypotheses of Theorem 3.3 are replaced by (4.1).

(b) We can now compare our Theorem 3.3 with the corresponding one in [20] in the case of Newton's method $\left(A(x)=F^{\prime}(x), G(x)=0(x \in \mathcal{D})\right)$.

Hypothesis (4.1) reduces to the famous Newton-Kantorovich hypothesis [1], [6], [15] for solving nonlinear equations:

$$
h_{K}=K \eta \leq 1 / 2,
$$

since $\sigma=K$ and $\mu_{0}=\mu_{1}=\ell=M=0$.

Note that in this case the functions $f_{m}(m \geq 1)$ should be defined by

$$
f_{m}(s)=\left(K s^{m-1}+2 L\left(1+s+s^{2}+\cdots+s^{m}\right)\right) \eta-2,
$$

and

$$
f_{m+1}(s)=f_{m}(s)+g(s) s^{m-1} \eta .
$$

But this time, the conditions corresponding to Lemma 3.1 should be

$$
\delta_{1}=\max \left\{\delta_{0} / 2, \delta_{+}\right\} \leq s_{\infty}=1-L \eta,
$$

whereas

$$
\delta=2 \delta_{1}
$$

Howeover, it is simple algebra to show that conditions (4.10)-(4.11) reduce to

$$
h_{A}=\bar{L} \eta \leq 1 / 2
$$

where

Note also that

$$
\bar{L}=\frac{1}{8}\left(K+4 L+\sqrt{K^{2}+8 K L}\right) .
$$

$$
L \leq K
$$

in general, and $K / L$ can be arbitrarily large.

In view of (4.9), (4.12) and (4.13), we get

$$
h_{K} \leq 1 / 2 \Rightarrow h_{A} \leq 1 / 2,
$$

but not necessarily vice versa unless $L=K$. 
In the example that follows, we show that $K / L$ can be arbitrarily large.

ExAmPle 4.3. Let $\mathcal{X}=\mathcal{Y}=\mathbb{R}, x_{0}=1$, and define scalar functions $F$ and $G$ by

$$
F(x)=c_{0} x+c_{1}+c_{2} \sin e^{c_{3} x}, \quad G(x)=0,
$$

where $c_{i}, i=0,1,2,3$, are given parameters. Using (4.15), it can easily be seen that for $c_{3}$ large and $c_{2}$ sufficiently small, $K / L$ can be arbitrarily large.

In the next examples, (4.1) is violated but (4.12) holds.

EXAmple 4.4. Let $\mathcal{X}=\mathcal{Y}=\mathbb{R}, x_{0}=1, U_{0}=\left\{x:\left|x-x_{0}\right| \leq 1-\beta\right\}$, $\beta \in[0,1 / 2)$, and define a function $F$ on $U_{0}$ by

$$
F(x)=x^{3}-\beta .
$$

Using the hypotheses of Theorem 3.3, we get

$$
\eta=\frac{1}{3}(1-\beta), \quad L=3-\beta, \quad K=2(2-\beta) .
$$

The Newton-Kantorovich condition (4.9) is violated, since

$$
\frac{4}{3}(1-\beta)(2-\beta)>1 \quad \text { for all } \beta \in[0,1 / 2) .
$$

Hence, there is no guarantee that (NTM) converges to $x^{\star}=\sqrt[3]{\beta}$, starting at $x_{0}=1$.

However, our condition (4.12) is true for all $\beta \in I=[.450339002,1 / 2)$. Hence, the conclusions of our Theorem 3.3 apply to equation (4.16) for all $\beta \in I$.

EXAmPLE 4.5. Let $\mathcal{X}=\mathcal{Y}=\mathcal{C}[0,1]$ be the space of real-valued continuous functions defined on the interval $[0,1]$ with norm

$$
\|x\|=\max _{0 \leq s \leq 1}|x(s)| .
$$

Let $\theta \in[0,1]$ be a given parameter. Consider the "cubic" integral equation

$$
u(s)=u^{3}(s)+\lambda u(s) \int_{0}^{1} q(s, t) u(t) d t+y(s)-\theta .
$$

Here the kernel $q(s, t)$ is a continuous function of two variables defined on $[0,1] \times[0,1]$; the parameter $\lambda$ is a real number called the albedo for scattering; $y(s)$ is a given continuous function defined on $[0,1]$; and $x(s)$ is the unknown function sought in $\mathcal{C}[0,1]$. Equations of the form (4.17) arise in the kinetic theory of gases [6], [8]. For simplicity, we choose $u_{0}(s)=y(s)=1$ and $q(s, t)=s /(s+t)$ for all $s, t \in[0,1]$ with $s+t \neq 0$. If we let $\mathcal{D}=U\left(u_{0}, 1-\theta\right)$, and define the operator $F$ on $\mathcal{D}$ by

$$
F(x)(s)=x^{3}(s)-x(s)+\lambda x(s) \int_{0}^{1} q(s, t) x(t) d t+y(s)-\theta
$$


for all $s \in[0,1]$, then every zero of $F$ satisfies equation (4.17). We have

$$
\max _{0 \leq s \leq 1}\left|\int \frac{s}{s+t} d t\right|=\ln 2 .
$$

Therefore, if we set $\xi=\left\|F^{\prime}\left(u_{0}\right)^{-1}\right\|$, then it follows from the hypotheses of Theorem 3.3 that

$$
\begin{gathered}
\eta=\xi(|\lambda| \ln 2+1-\theta), \\
K=2 \xi(|\lambda| \ln 2+3(2-\theta)), \quad L=\xi(2|\lambda| \ln 2+3(3-\theta)) .
\end{gathered}
$$

It follows from Theorem 3.3 that if condition (4.12) holds, then problem (4.17) has a unique solution near $u_{0}$. This assumption is weaker than the one given before using the Newton-Kantorovich hypothesis (4.9).

Note also that $L<K$ for all $\theta \in[0,1]$.

EXAMPLE 4.6. Consider the following nonlinear boundary value problem [6]:

$$
\left\{\begin{array}{l}
u^{\prime \prime}=-u^{3}-\gamma u^{2} \\
u(0)=0, \quad u(1)=1
\end{array}\right.
$$

It is well known that this problem can be formulated as the integral equation

$$
u(s)=s+\int_{0}^{1} Q(s, t)\left(u^{3}(t)+\gamma u^{2}(t)\right) d t
$$

where $Q$ is the Green function:

$$
Q(s, t)= \begin{cases}t(1-s) & t \leq s \\ s(1-t) & s<t\end{cases}
$$

We observe that

$$
\max _{0 \leq s \leq 1} \int_{0}^{1}|Q(s, t)|=\frac{1}{8} .
$$

Let $\mathcal{X}=\mathcal{Y}=\mathcal{C}[0,1]$ with norm

$$
\|x\|=\max _{0 \leq s \leq 1}|x(s)| .
$$

Then problem (4.19) is in the form (1.1), where $F: \mathcal{D} \rightarrow \mathcal{Y}$ is defined as

$$
[F(x)](s)=x(s)-s-\int_{0}^{1} Q(s, t)\left(x^{3}(t)+\gamma x^{2}(t)\right) d t,
$$

and

$$
G(x)(s)=0 .
$$

It is easy to verify that the Fréchet derivative of $F$ is

$$
\left[F^{\prime}(x) v\right](s)=v(s)-\int_{0}^{1} Q(s, t)\left(3 x^{2}(t)+2 \gamma x(t)\right) v(t) d t .
$$


If we set $u_{0}(s)=s$, and $\mathcal{D}=U\left(u_{0}, R\right)$, then since $\left\|u_{0}\right\|=1$, it is easy to verify that $U\left(u_{0}, R\right) \subset U(0, R+1)$. It follows that if $2 \gamma<5$, then

$$
\begin{aligned}
\left\|I-F^{\prime}\left(u_{0}\right)\right\| & \leq \frac{3\left\|u_{0}\right\|^{2}+2 \gamma\left\|u_{0}\right\|}{8}=\frac{3+2 \gamma}{8}, \\
\left\|F^{\prime}\left(u_{0}\right)^{-1}\right\| & \leq \frac{1}{1-\frac{3+2 \gamma}{8}}=\frac{8}{5-2 \gamma}, \\
\left\|F\left(u_{0}\right)\right\| & \leq \frac{\left\|u_{0}\right\|^{3}+\gamma\left\|u_{0}\right\|^{2}}{8}=\frac{1+\gamma}{8}, \\
\left\|F\left(u_{0}\right)^{-1} F\left(u_{0}\right)\right\| & \leq \frac{1+\gamma}{5-2 \gamma} .
\end{aligned}
$$

On the other hand, for $x, y \in \mathcal{D}$, we have

$$
\left[\left(F^{\prime}(x)-F^{\prime}(y)\right) v\right](s)=-\int_{0}^{1} Q(s, t)\left(3 x^{2}(t)-3 y^{2}(t)+2 \gamma(x(t)-y(t))\right) v(t) d t .
$$

Consequently,

$$
\begin{aligned}
\left\|F^{\prime}(x)-F^{\prime}(y)\right\| & \leq \frac{\|x-y\|(2 \gamma+3(\|x\|+\|y\|))}{8} \\
& \leq \frac{\|x-y\|\left(2 \gamma+6 R+6\left\|u_{0}\right\|\right)}{8}=\frac{\gamma+6 R+3}{4}\|x-y\|, \\
\left\|F^{\prime}(x)-F^{\prime}\left(u_{0}\right)\right\| & \leq \frac{\left\|x-u_{0}\right\|\left(2 \gamma+3\left(\|x\|+\left\|u_{0}\right\|\right)\right)}{8} \\
& \leq \frac{\left\|x-u_{0}\right\|\left(2 \gamma+3 R+6\left\|u_{0}\right\|\right)}{8}=\frac{2 \gamma+3 R+6}{8}\left\|x-u_{0}\right\| .
\end{aligned}
$$

Therefore, the conditions of Theorem 3.3 hold with

$$
\eta=\frac{1+\gamma}{5-2 \gamma}, \quad K=\frac{\gamma+6 R+3}{4}, \quad L=\frac{2 \gamma+3 R+6}{8} .
$$

Note also that $L<K$.

Lemma 4.7. Assume there exist constants $L, K, \eta \geq 0$ such that

$$
h_{A}=\bar{L} \eta \leq 1 / 2,
$$

where

$$
\bar{L}=\frac{1}{8}\left(K+4 L+\sqrt{K^{2}+8 L K}\right) .
$$

The inequality in (4.20) is strict if $L=0$. Then the sequence $\left\{t_{k}\right\}(k \geq 0)$ given by

$$
t_{0}=0, \quad t_{1}=\eta, \quad t_{k+1}=t_{k}+\frac{L_{1}\left(t_{k}-t_{k-1}\right)^{2}}{2\left(1-L t_{k}\right)} \quad(k \geq 1)
$$

is well defined, nondecreasing, bounded above by $t^{\star \star}$, and converges to its 
least upper bound $t^{\star} \in\left[0, t^{\star \star}\right]$, where

$$
\begin{gathered}
L_{1}= \begin{cases}L & \text { if } k=1, \\
K & \text { if } k>1,\end{cases} \\
t^{\star \star}=\frac{2 \eta}{2-\delta}, \\
1 \leq \delta=\frac{4 K}{K+\sqrt{K^{2}+8 L K}}<2 \quad \text { for } L \neq 0 .
\end{gathered}
$$

Moreover, the following estimates hold:

$$
\begin{aligned}
L t^{\star}<1, & \\
0 \leq t_{k+1}-t_{k} & \leq \frac{\delta}{2}\left(t_{k}-t_{k-1}\right) \leq \cdots \leq\left(\frac{\delta}{2}\right)^{k} \eta \quad(k \geq 1), \\
t_{k+1}-t_{k} & \leq\left(\frac{\delta}{2}\right)^{k}\left(2 h_{A}\right)^{2^{k}-1} \eta \quad(k \geq 0), \\
0 \leq t^{\star}-t_{k} & \leq\left(\frac{\delta}{2}\right)^{k} \frac{\left(2 h_{A}\right)^{2^{k}-1} \eta}{1-\left(2 h_{A}\right)^{2^{k}}} \quad\left(2 h_{A}<1, k \geq 0\right) .
\end{aligned}
$$

REMARK 4.8. Under the Newton-Kantorovich condition (A.15), the majorizing sequence

$$
\overline{\bar{t}}_{0}=0, \quad \overline{\bar{t}}_{1}=\eta, \quad \overline{\bar{t}}_{k+1}=\overline{\bar{t}}_{k}+\frac{K\left(\overline{\bar{t}}_{k}-\overline{\bar{t}}_{k-1}\right)^{2}}{2\left(1-L \overline{\bar{t}}_{k}\right)} \quad(k \geq 1)
$$

was used in [10], [11], [15], [18]-[22]. The corresponding ratio (see (4.27)) is given by

$$
2 h_{K}=K \eta
$$

But we have

$$
h_{A}<h_{K}
$$

provided that $L<K$. Hence, the sequence $\left\{t_{n}\right\}$ given in Lemma 4.7 is a tighter majorizing sequence than $\left\{\overline{\bar{t}}_{n}\right\}$, obtained under weaker sufficient convergence conditions (see (A.18)).

Application 4.9. Let

$$
A\left(y_{n}\right)=F^{\prime}\left(y_{n}\right)+\left[y_{n-1}, y_{n} ; G\right] \quad(n \geq 0)
$$

and consider (NTM) in the form

$$
\text { (4.29) } y_{n+1}=y_{n}-\left(F^{\prime}\left(y_{n}\right)+\left[y_{n-1}, y_{n} ; G\right]\right)^{-1}\left(F\left(y_{n}\right)+G\left(y_{n}\right)\right) \quad(n \geq 0) \text {. }
$$

This method has order $(1+\sqrt{5}) / 2$ (see [6]) (the same as the method of chord), but higher than the order of

$$
z_{n+1}=z_{n}-F^{\prime}\left(z_{n}\right)^{-1}\left(F\left(z_{n}\right)+G\left(z_{n}\right)\right) \quad(n \geq 0)
$$


considered in [9], [20]-[22], and the method of chord

$$
w_{n+1}=w_{n}-\left[w_{n-1}, w_{n} ; G\right]^{-1}\left(F\left(w_{n}\right)+G\left(w_{n}\right)\right) \quad(n \geq 0),
$$

where $[x, y ; G]$ denotes the divided difference of $G$ at the points $x$ and $y[6]$.

Let us provide an example for this case.

Example 4.10. Let $X=Y=\left(\mathbb{R}^{2},\|\cdot\|_{\infty}\right)$. Consider the system

$$
3 x^{2} y+y^{2}-1+|x-1|=0, \quad x^{4}+x y^{3}-1+|y|=0 .
$$

Set $\|x\|_{\infty}=\left\|\left(x^{\prime}, x^{\prime \prime}\right)\right\|_{\infty}=\max \left\{\left|x^{\prime}\right|,\left|x^{\prime \prime}\right|\right\}, F=\left(F_{1}, F_{2}\right), G=\left(G_{1}, G_{2}\right)$. For $x=\left(x^{\prime}, x^{\prime \prime}\right) \in \mathbb{R}^{2}$ we take $F_{1}\left(x^{\prime}, x^{\prime \prime}\right)=3\left(x^{\prime}\right)^{2} x^{\prime \prime}+\left(x^{\prime \prime}\right)^{2}-1, F_{2}\left(x^{\prime}, x^{\prime \prime}\right)=$ $\left(x^{\prime}\right)^{4}+x^{\prime}\left(x^{\prime \prime}\right)^{3}-1, G_{1}\left(x^{\prime}, x^{\prime \prime}\right)=\left|x^{\prime}-1\right|, G_{2}\left(x^{\prime}, x^{\prime \prime}\right)=\left|x^{\prime \prime}\right|$. We shall take $[x, y ; G] \in M_{2 \times 2}(\mathbb{R})$ as

$$
[x, y ; G]_{i, 1}=\frac{G_{i}\left(y^{\prime}, y^{\prime \prime}\right)-G_{i}\left(x^{\prime}, y^{\prime \prime}\right)}{y^{\prime}-x^{\prime}}, \quad[x, y ; G]_{i, 2}=\frac{G_{i}\left(x^{\prime}, y^{\prime \prime}\right)-G_{i}\left(x^{\prime}, x^{\prime \prime}\right)}{y^{\prime \prime}-x^{\prime \prime}}
$$

$i=1,2$, provided that $y^{\prime} \neq x^{\prime}$ and $y^{\prime \prime} \neq x^{\prime \prime}$. Otherwise define $[x, y ; G]$ to be the zero matrix in $M_{2 \times 2}(\mathbb{R})$.

Using method (4.30) with $z_{0}=(1,0)$ we obtain:

\begin{tabular}{llll}
\hline$n$ & $z_{n}^{(1)}$ & $z_{n}^{(2)}$ & $\left\|z_{n}-z_{n-1}\right\|$ \\
\hline 0 & 1 & 0 & \\
1 & 1 & 0.333333333333333 & $3.333 \mathrm{E}-1$ \\
2 & 0.906550218340611 & 0.354002911208151 & $9.344 \mathrm{E}-2$ \\
3 & 0.885328400663412 & 0.338027276361322 & $2.122 \mathrm{E}-2$ \\
4 & 0.891329556832800 & 0.326613976593566 & $1.141 \mathrm{E}-2$ \\
5 & 0.895238815463844 & 0.326406852843625 & $3.909 \mathrm{E}-3$ \\
6 & 0.895154671372635 & 0.327730334045043 & $1.323 \mathrm{E}-3$ \\
7 & 0.894673743471137 & 0.327979154372032 & $4.809 \mathrm{E}-4$ \\
8 & 0.894598908977448 & 0.327865059348755 & $1.140 \mathrm{E}-4$ \\
9 & 0.894643228355865 & 0.327815039208286 & $5.002 \mathrm{E}-5$ \\
10 & 0.894659993615645 & 0.327819889264891 & $1.676 \mathrm{E}-5$ \\
11 & 0.894657640195329 & 0.327826728208560 & $6.838 \mathrm{E}-6$ \\
12 & 0.894655219565091 & 0.327827351826856 & $2.420 \mathrm{E}-6$ \\
13 & 0.894655074977661 & 0.327826643198819 & $7.086 \mathrm{E}-7$ \\
$\ldots$ & & & \\
39 & 0.894655373334687 & 0.327826521746298 & $5.149 \mathrm{E}-19$ \\
\hline
\end{tabular}

Using the method of chord (i.e., (4.31)) with $w_{-1}=(1,0)$, and $w_{0}=(5,5)$, we obtain: 


\begin{tabular}{rlll}
\hline$n$ & $w_{n}^{(1)}$ & $w_{n}^{(2)}$ & $\left\|w_{n}-w_{n-1}\right\|$ \\
\hline-1 & 5 & 5 & \\
0 & 1 & 0 & $5.000 \mathrm{E}+00$ \\
1 & 0.989800874210782 & 0.012627489072365 & $1.262 \mathrm{E}-02$ \\
2 & 0.921814765493287 & 0.307939916152262 & $2.953 \mathrm{E}-01$ \\
3 & 0.900073765669214 & 0.325927010697792 & $2.174 \mathrm{E}-02$ \\
4 & 0.894939851625105 & 0.327725437396226 & $5.133 \mathrm{E}-03$ \\
5 & 0.894658420586013 & 0.327825363500783 & $2.814 \mathrm{E}-04$ \\
6 & 0.894655375077418 & 0.327826521051833 & $3.045 \mathrm{E}-04$ \\
7 & 0.894655373334698 & 0.327826521746293 & $1.742 \mathrm{E}-09$ \\
8 & 0.894655373334687 & 0.327826521746298 & $1.076 \mathrm{E}-14$ \\
9 & 0.894655373334687 & 0.327826521746298 & $5.421 \mathrm{E}-20$ \\
\hline
\end{tabular}

Using our method (4.29) with $y_{-1}=(1,0), y_{0}=(5,5)$, we obtain

\begin{tabular}{rlll}
\hline$n$ & $y_{n}^{(1)}$ & $y_{n}^{(2)}$ & $\left\|y_{n}-y_{n-1}\right\|$ \\
\hline-1 & 5 & 5 & \\
0 & 1 & 0 & 5 \\
1 & 0.909090909090909 & 0.363636363636364 & $3.636 \mathrm{E}-01$ \\
2 & 0.894886945874111 & 0.329098638203090 & $3.453 \mathrm{E}-02$ \\
3 & 0.894655531991499 & 0.327827544745569 & $1.271 \mathrm{E}-03$ \\
4 & 0.894655373334793 & 0.327826521746906 & $1.022 \mathrm{E}-06$ \\
5 & 0.894655373334687 & 0.327826521746298 & $6.089 \mathrm{E}-13$ \\
6 & 0.894655373334687 & 0.327826521746298 & $2.710 \mathrm{E}-20$ \\
\hline
\end{tabular}

The solution is

$$
x^{\star}=(.894655373334687, .327826521746298)
$$

chosen from the lists of the tables displayed above.

Hence method (4.29) converges faster than (4.30) suggested in Chen and Yamamoto [9], Zabrejko and Nguen [21] in this case, and the method of chord [6].

\section{Appendix}

Proof of Lemma 2.1. We shall show (2.21) and (2.22) by induction on $n$. These estimates hold for $n=0$ by (2.2), (2.3), (2.10), (2.11), (2.15)-(2.18).

Assume that (2.21) and (2.22) hold for all $k \leq n$. Then

$$
\begin{aligned}
s_{k+1} & \leq t_{k+1}+\frac{\delta}{2}\left(s_{k}-t_{k}\right) \leq s_{k}+\frac{\delta}{2}\left(s_{k}-t_{k}\right)+\frac{\delta}{2}\left(s_{k}-t_{k}\right) \\
& \leq s_{k}+2\left(\frac{\delta}{2}\right)^{k+1} \eta \leq s_{k-1}+2\left(\frac{\delta}{2}\right)^{k} \eta+2\left(\frac{\delta}{2}\right)^{k+1} \eta
\end{aligned}
$$




$$
\begin{aligned}
& \leq s_{0}+2\left\{\frac{\delta}{2}+\cdots+\left(\frac{\delta}{2}\right)^{k+1}\right\} \eta=\eta+2 \frac{\delta}{2}\left\{1+\cdots+\left(\frac{\delta}{2}\right)^{k}\right\} \eta \\
& =\left\{1+\frac{1-(\delta / 2)^{k+1}}{1-\delta / 2} \delta\right\} \eta \leq t^{\star \star} \quad \text { by }(2.19),
\end{aligned}
$$

and

(A.2)

$$
\begin{aligned}
t_{k+1} & \leq s_{k}+\frac{\delta}{2}\left(s_{k}-t_{k}\right) \\
& \leq\left\{1+\frac{1-(\delta / 2)^{k}}{1-\delta / 2} \delta+\left(\frac{\delta}{2}\right)^{k+1}\right\} \eta \leq t^{\star \star} \quad \text { by }(2.19) .
\end{aligned}
$$

Estimate (2.21) certainly holds if

$$
t_{k+1}-s_{k} \leq \frac{\delta}{2}\left(s_{k}-t_{k}\right)
$$

or

$$
\begin{gathered}
\frac{\alpha^{2} \gamma K}{2}\left(s_{k}-t_{k}\right)+\alpha \gamma\left(M t_{k}+b\right) \leq \frac{\delta}{2}\left(1-\ell-L t_{k}\right) \\
L t_{k}+\ell<1
\end{gathered}
$$

Estimates (A.3) and (A.4) in turn hold if

$$
\begin{aligned}
& \frac{\alpha^{2} \gamma K}{2}\left(\frac{\delta}{2}\right)^{k} \eta+\alpha \gamma M\left\{1+\frac{1-(\delta / 2)^{k-1}}{1-\delta / 2} \delta+\left(\frac{\delta}{2}\right)^{k}\right\} \eta \\
& \quad+L \frac{\delta}{2}\left\{1+\frac{1-(\delta / 2)^{k-1}}{1-\delta / 2} \delta+\left(\frac{\delta}{2}\right)^{k}\right\} \eta-\frac{\delta}{2}(1-\ell)+\alpha \gamma b \leq 0 .
\end{aligned}
$$

Estimate (A.5) motivates us to define functions $f_{k}$ given by $(2.4)$ for $w=$ $\delta / 2$, and show instead of (A.5):

$$
f_{k}(\delta) \leq 0 \quad(k \geq 1) .
$$

By letting $k \rightarrow \infty$ in (A.5), we get

or

$$
\alpha \gamma M\left(1+\frac{2 w}{1-w}\right) \eta+L w\left(1+\frac{2 w}{1-w}\right) \eta-(1-\ell) w+\alpha \gamma b=0
$$

$$
f_{\infty}\left(w_{\infty}\right)=0
$$

By hypothesis, we also have $f_{2}\left(w_{2}\right)=0$.

We need to find a relationship between two consecutive $f_{k}$ :

$$
\begin{aligned}
f_{k+1}(w)= & f_{k}(w)+\frac{\alpha^{2} \gamma K}{2} w^{k+1} \eta-\frac{\alpha^{2} \gamma K}{2} w^{k} \eta \\
& +\alpha \gamma M\left(w^{k}+w^{k+1}\right) \eta+L w\left(w^{k}+w^{k+1}\right) \eta \\
= & f_{k}(w)+g(w) w^{k} \eta,
\end{aligned}
$$

where $g$ is given by (2.7). 
Using (A.7) for $k=2$, we get

$$
f_{3}\left(w_{2}\right)=f_{2}\left(w_{2}\right)+g\left(w_{2}\right) w_{2}^{2} \eta=g\left(w_{2}\right) w_{2}^{2} \eta \leq 0,
$$

since $f_{2}\left(w_{2}\right)=0$ and $g\left(w_{2}\right) \leq 0$ (by $\left.(2.16)\right)$.

We also have

$$
f_{3}(0)=\alpha \gamma(M \eta+b) \geq 0 .
$$

It follows from (A.8), (A.9), and the intermediate value theorem that there exists $w_{3} \in\left[0, w_{2}\right]$ such that $f_{3}\left(w_{3}\right)=0$. Denote the minimal zero of $f_{3}$ in $\left[0, w_{2}\right]$ by the same symbol $w_{3}$.

Assume that there exists a minimal $w_{k} \in\left[0, w_{k-1}\right]$ with $f_{k}\left(w_{k}\right)=0$. As in (A.8), we get

$$
\begin{aligned}
f_{k+1}(0) & =\alpha \gamma(M \eta+b) \geq 0, \\
f_{k+1}\left(w_{k}\right) & =f_{k}\left(w_{k}\right)+g\left(w_{k}\right) w_{k}^{k} \eta \leq 0 .
\end{aligned}
$$

since $f_{k}\left(w_{k}\right)=0$, and $g\left(w_{k}\right) \leq 0$ (by $\left.(2.16)\right)$.

Hence, again we deduce that there exists a minimal $w_{k+1} \in\left[0, w_{k}\right]$ such that $f_{k+1}\left(w_{k+1}\right)=0$.

The sequence $\left\{w_{k}\right\}$ is nonincreasing, bounded below by zero, and converges to its greatest lower bound $w^{\star \star}$ satisfying $w^{\star \star} \geq w_{\infty}$. It then follows by (2.18) that (A.6) holds.

Using the induction hypotheses, estimate (2.22) will hold if

$$
\begin{gathered}
0 \leq s_{n+1}-t_{n+1} \leq \frac{\delta}{2}\left(s_{n}-t_{n}\right), \\
L t_{n+1}+\ell<1 .
\end{gathered}
$$

These estimates hold for $n=0$ by the initial conditions.

Estimates (A.11) and (A.12) will also hold if

$$
\begin{aligned}
& \frac{1}{1-\ell-L t_{k+1}}\left\{\frac{K}{2}\left(\left(t_{k+1}-s_{k}\right)+\left(s_{k}-t_{k}\right)\right)^{2}\right. \\
& \quad+\left(M t_{k}+b\right)\left(\left(t_{k+1}-s_{k}\right)+\left(s_{k}-t_{k}\right)\right) \\
& \left.\quad+\frac{\alpha^{2} \gamma K}{2}\left(s_{k}-t_{k}\right)^{2}+\alpha \gamma\left(M t_{k}+\mu\right)\left(s_{k}-t_{k}\right)+N \alpha \gamma\left(s_{k}-t_{k}\right)\right\} \\
& \leq \frac{\delta}{2}\left(s_{k}-t_{k}\right)
\end{aligned}
$$


or

$$
\begin{aligned}
& \frac{1}{1-\ell-L t_{k+1}}\left\{\frac{K}{2}\left(1+\frac{\delta}{2}\right)^{2}\left(s_{k}-t_{k}\right)^{2}\right. \\
& \quad+\left(M t_{k}+b\right)\left(1+\frac{\delta}{2}\right)\left(s_{k}-t_{k}\right) \\
& \left.\quad+\frac{\alpha^{2} \gamma K}{2}\left(s_{k}-t_{k}\right)^{2}+\alpha \gamma\left(M t_{k}+\mu\right)\left(s_{k}-t_{k}\right)+N \alpha \gamma\left(s_{k}-t_{k}\right)\right\} \\
& \leq \frac{\delta}{2}\left(s_{k}-t_{k}\right)
\end{aligned}
$$

or

$$
\begin{aligned}
& \frac{K}{2}\left(1+\frac{\delta}{2}\right)^{2}\left(s_{k}-\right.\left.t_{k}\right)+\left(M t_{k}+b\right)\left(1+\frac{\delta}{2}\right)+\frac{\alpha^{2} \gamma K}{2}\left(s_{k}-t_{k}\right) \\
&+\alpha \gamma\left(M t_{k}+\mu\right)+N \alpha \gamma+L \frac{\delta}{2} t_{k+1}-(1-\ell) \frac{\delta}{2} \leq 0
\end{aligned}
$$

or

(A.14)

$$
\begin{aligned}
& \frac{K}{2}\left(1+\frac{\delta}{2}\right)^{2}\left(\frac{\delta}{2}\right)^{k} \eta+\left(c+\frac{\delta}{2}\right)\left\{M\left(1+\frac{1-(\delta / 2)^{k-1}}{1-\delta / 2} \delta+\left(\frac{\delta}{2}\right)^{k}\right) \eta+b\right\} \\
+ & \frac{\alpha^{2} \gamma K}{2}\left(\frac{\delta}{2}\right)^{k} \eta+L \frac{\delta}{2} M\left\{1+\frac{1-(\delta / 2)^{k}}{1-\delta / 2} \delta+\left(\frac{\delta}{2}\right)^{k+1}\right\} \eta-(1-\ell) \frac{\delta}{2} \leq 0 .
\end{aligned}
$$

Let again $w=\delta / 2$, and consider the functions $f_{k}^{1}$ given by (2.5). Then (A.14) will hold if

$$
f_{k}^{1}(\delta) \leq 0 \quad(k \geq 1) .
$$

By letting $k \rightarrow \infty$ in (A.14), we get

$$
(c+w)\left(b+\frac{M w \eta}{1-w}\right)+\frac{L M w^{2} \eta}{1-w}-(1-\ell) w=0
$$

if $f_{\infty}^{1}\left(w_{\infty}^{1}\right)=0$, which is true by hypothesis.

We need to find a relationship between two consecutive $f_{k}^{1}$ :

$$
\begin{aligned}
f_{k+1}^{1}(w)= & f_{k}^{1}(w)+\frac{K}{2}(1+w)^{2} w^{k+1} \eta-\frac{K}{2}(1+w)^{2} w^{k} \eta \\
& +(c+w) M\left(w^{k}+w^{k+1}\right) \eta+\frac{\alpha^{2} \gamma K}{2}\left(w^{k+1}-w^{k}\right) \eta \\
& +L M w\left(w^{k+1}+w^{k+2}\right) \eta \\
= & f_{k}^{1}(w)+g^{1}(w) w^{k} \eta
\end{aligned}
$$

where $g^{1}$ is given by $(2.9)$. 
Using (A.17) for $k=2$, we get

$$
f_{3}\left(w_{2}^{1}\right)=f_{2}^{1}\left(w_{2}^{1}\right)+g^{1}\left(w_{2}^{1}\right)\left(w_{2}^{1}\right)^{2} \eta=g^{1}\left(w_{2}^{1}\right)\left(w_{2}^{1}\right)^{2} \eta \leq 0,
$$

since $f_{2}^{1}\left(w_{2}^{1}\right)=0$, and $g^{1}\left(w_{2}^{1}\right) \leq 0$ (by $\left.(2.17)\right)$.

We also have

$$
f_{3}^{1}(0)=c(M \eta+b) \geq 0 .
$$

Hence, there exists $w_{3}^{1} \in\left[0, w_{2}^{1}\right]$ such that $f_{3}^{1}\left(w_{3}^{1}\right)=0$. Denote the minimal zero of $f_{3}^{1}$ in $\left[0, w_{2}^{1}\right]$ by the same symbol $w_{3}^{1}$.

Assume that there exists a minimal $w_{k}^{1} \in\left[0, w_{k-1}^{1}\right]$ with $f_{k}^{1}\left(w_{k}^{1}\right)=0$. As in (A.18), we get

$$
\begin{aligned}
f_{k+1}^{1}(0) & =c(M \eta+b) \geq 0, \\
f_{k+1}^{1}\left(w_{k}^{1}\right) & =f_{k}^{1}\left(w_{k}^{1}\right)+g^{1}\left(w_{k}^{1}\right)\left(w_{k}^{1}\right)^{k} \eta \leq 0,
\end{aligned}
$$

which implies the existence of $w_{k+1}^{1} \in\left[0, w_{k}^{1}\right]$ such that $f_{k+1}^{1}\left(w_{k+1}^{1}\right)=0$.

The sequence $\left\{w_{k}^{1}\right\}$ is nonincreasing, bounded below by zero, and as such it converges to its infinimum $w_{\star \star}$ satisfying $w_{\star \star} \geq w_{\infty}^{1}$. It then follows from (2.18) that (A.15) holds.

The induction for (2.22) is thus complete. It then follows from (2.21) and (2.22) that the sequences $\left\{t_{n}\right\},\left\{s_{n}\right\}$ are nondecreasing, bounded above by $t^{\star \star}$, with $t_{n} \leq s_{n} \leq t_{n+1} \leq s_{n+1} \leq t^{\star \star}$, and as such they converge to their common least upper bound $t^{\star} \in\left[0, t^{\star \star}\right]$.

We also have, for $m \geq 2$,

$$
\begin{aligned}
s_{n+m}-t_{n}= & \left(s_{n+m}-t_{n+m}\right)+\left(t_{n+m}-t_{n}\right) \\
= & \left(s_{n+m}-t_{n+m}\right)+\left(t_{n+m}-s_{n+m-1}\right)+\left(s_{n+m-1}-t_{n}\right) \\
\leq & \left(\frac{\delta}{2}\right)^{n+m} \eta+\left(\frac{\delta}{2}\right)^{n+m} \eta+\left(\frac{\delta}{2}\right)^{n+m-1} \eta+\left(\frac{\delta}{2}\right)^{n+m-1} \eta+\cdots \\
& +\left(\frac{\delta}{2}\right)^{n+1} \eta+\left(\frac{\delta}{2}\right)^{n+1} \eta+\left(\frac{\delta}{2}\right)^{n} \eta \\
= & 2\left(\frac{\delta}{2}\right)^{n+1} \eta\left\{1+\frac{\delta}{2}+\cdots+\left(\frac{\delta}{2}\right)^{m-2}\right\}+\left(\frac{\delta}{2}\right)^{n} \eta \\
= & 2 \eta\left(\frac{\delta}{2}\right)^{n+1} \frac{1-(\delta / 2)^{m-1}}{1-\delta / 2}+\left(\frac{\delta}{2}\right)^{n} \eta .
\end{aligned}
$$

By letting $m \rightarrow \infty$ in (A.21), we obtain (2.23). 
We also have

(A.22)

$$
\begin{aligned}
t_{n+m}-s_{n}= & \left(t_{n+m}-s_{n+m-1}\right)+\left(s_{n+m-1}-t_{n+m-1}\right)+\left(t_{n+m-1}-s_{n}\right) \\
\leq & \left(\frac{\delta}{2}\right)^{n+m} \eta+\left(\frac{\delta}{2}\right)^{n+m-1} \eta+\left(\frac{\delta}{2}\right)^{n+m-1} \eta+\left(\frac{\delta}{2}\right)^{n+m-2} \eta \\
& +\left(\frac{\delta}{2}\right)^{n+m-2} \eta+\cdots+\left(\frac{\delta}{2}\right)^{n+1} \eta+\left(\frac{\delta}{2}\right)^{n+1} \eta \\
= & \left(\frac{\delta}{2}\right)^{n+2} \eta\left\{1+\frac{\delta}{2}+\cdots+\left(\frac{\delta}{2}\right)^{m-2}\right\} \\
& +\left(\frac{\delta}{2}\right)^{n+1} \eta\left\{1+\frac{\delta}{2}+\cdots+\left(\frac{\delta}{2}\right)^{m-2}\right\}+\left(\frac{\delta}{2}\right)^{n+1} \eta \\
= & \left(\frac{\delta}{2}\right)^{n+2} \eta \frac{1-(\delta / 2)^{m-1}}{1-\delta / 2}+\left(\frac{\delta}{2}\right)^{n+1} \eta \frac{1-(\delta / 2)^{m-2}}{1-\delta / 2}+\left(\frac{\delta}{2}\right)^{n+1} \eta .
\end{aligned}
$$

By letting $m \rightarrow \infty$ in (A.21), we obtain (2.24).

That completes the proof of Lemma 2.1.

Proof of Lemma 3.1. We shall show by induction on $m$ that

$$
\begin{aligned}
0<t_{m+2}-t_{m+1} & =\frac{K\left(t_{m+1}-t_{m}\right)+2\left(M t_{m}+\mu\right)}{2\left(1-L t_{m+1}-\ell\right)}\left(t_{m+1}-t_{m}\right) \\
& \leq \frac{\delta}{2}\left(t_{m+1}-t_{m}\right)
\end{aligned}
$$

$$
\ell+L t_{m+1}<1
$$

If (A.23) and (A.24) hold, then (3.12) holds, and

$$
\begin{aligned}
t_{m+2} & \leq t_{m+1}+\frac{\delta}{2}\left(t_{m+1}-t_{m}\right) \\
& \leq t_{m}+\frac{\delta}{2}\left(t_{m}-t_{m-1}\right)+\frac{\delta}{2}\left(t_{m+1}-t_{m}\right) \\
& \leq \eta+\left(\frac{\delta}{2}\right) \eta+\cdots+\left(\frac{\delta}{2}\right)^{m+1} \eta \\
& =\frac{1-(\delta / 2)^{m+2}}{1-\delta / 2} \eta<\frac{2 \eta}{2-\delta}=t^{\star \star} \quad \text { by (3.11). }
\end{aligned}
$$

It will then also follow that the sequence $\left\{t_{m}\right\}$ is increasing, bounded above by $t^{\star \star}$, and as such it will converge to some $t^{\star} \in\left[0, t^{\star \star}\right]$.

Estimates (A.23) and (A.24) hold by the initial conditions for $m=0$. Indeed, (A.23) and (A.24) become 


$$
\begin{aligned}
0<t_{2}-t_{1} & =\frac{K\left(t_{1}-t_{0}\right)+2\left(M t_{0}+\mu\right)}{2\left(1-L t_{1}-\ell\right)}\left(t_{1}-t_{0}\right) \\
& =\frac{K \eta+2 \mu}{2(1-L \eta-\ell)}\left(t_{1}-t_{0}\right)=\frac{\delta_{0}}{2}\left(t_{1}-t_{0}\right) \leq \frac{\delta}{2}\left(t_{1}-t_{0}\right), \\
L \eta+\ell<1, &
\end{aligned}
$$

which are true by the choice of $\delta_{0}, \delta,(3.3),(3.10)$, and the initial conditions. Let us assume (A.23)-(A.24) hold for all $m \leq n+1$.

Estimate (A.23) can be rewritten as

$$
K\left(t_{m+1}-t_{m}\right)+2\left(M t_{m}+\mu\right) \leq\left(1-L t_{m+1}-\ell\right) \delta,
$$

or

$$
K\left(t_{m+1}-t_{m}\right)+2\left(M t_{m}+\mu\right)+\delta L t_{m+1}+\delta \ell-\ell \leq 0,
$$

or

$$
\begin{aligned}
& K\left(\frac{\delta}{2}\right)^{m} \eta+2\left(M \frac{1-(\delta / 2)^{m}}{1-\delta / 2} \eta+\mu\right) \\
& +\delta L \frac{1-(\delta / 2)^{m+1}}{1-\delta / 2} \eta+\delta(\ell-1) \leq 0 .
\end{aligned}
$$

Replace $\delta / 2$ by $s$, and define functions $f_{m}$ on $[0,+\infty)(m \geq 1)$ by

$$
\begin{aligned}
f_{m}(s)= & K s^{m} \eta+2\left[M\left(1+s+s^{2}+\cdots+s^{m-1}\right) \eta+\mu\right] \\
& +2 s L\left(1+s+\cdots+s^{m}\right) \eta+2 s(\ell-1) .
\end{aligned}
$$

Estimate (A.27) certainly holds if

$$
f_{m}(\delta) \leq 0 \quad(m \geq 1)
$$

We need to find a relationship between two consecutive $f_{m}$ :

$$
\begin{aligned}
f_{m+1}(s)= & K s^{m+1} \eta+2\left(M\left(1+s+s^{2}+\cdots+s^{m-1}+s^{m}\right) \eta+\mu\right) \\
& +2 s L\left(1+s+\cdots+s^{m}+s^{m+1}\right) \eta+2 s(\ell-1) \\
= & K s^{m+1} \eta-K s^{m} \eta+K s^{m} \eta \\
& +2\left(M\left(1+s+s^{2}+\cdots+s^{m-1}\right) \eta+\mu\right) \\
& +2 M s^{m} \eta+2 s L\left(1+s+\cdots+s^{m}\right) \eta+2 s L s^{m+1} \eta+2 s(\ell-1) \\
= & f_{m}(s)+K s^{m+1} \eta-K s^{m} \eta+2 M s^{m} \eta+2 s L s^{m+1} \eta \\
= & f_{m}(s)+g(s) s^{m} \eta,
\end{aligned}
$$

where

$$
g(s)=2 L s^{2}+K s+2 M-K \text {. }
$$


Note that in view of (3.2), the function $g$ has a positive zero $\delta_{+}$given by (3.5), and

$$
g(s)<0, \quad s \in\left(0, \delta_{+}\right) .
$$

By hypothesis, the function $f_{1}$ has a minimal positive zero $s_{1}$. Using (3.2), it is simple algebra to show $s_{1} \in[0,1)$. It then follows from (A.30) and (A.31) that

$$
f_{2}\left(s_{1}\right)=f_{1}\left(s_{1}\right)+g\left(s_{1}\right) s_{1}^{m} \eta=g\left(s_{1}\right) s_{1}^{m} \eta<0,
$$

since $f_{1}\left(s_{1}\right)=0$ and $g\left(s_{1}\right)<0$. We also have, from (A.28),

$$
f_{m}(0)=2(M \eta+\mu)>0 \quad(m \geq 1) .
$$

It follows from the intermediate value theorem that there exists a minimal $s_{2} \in\left(0, s_{1}\right)$ such that $f_{2}\left(s_{2}\right)=0$. Assume that there exists $s_{m} \in\left(0, s_{m-1}\right)$ with $f_{m}\left(s_{m}\right)=0$. As in (A.33) we have

$$
f_{m+1}\left(s_{m}\right)=f_{m}\left(s_{m}\right)+g\left(s_{m}\right) s_{m}^{m} \eta<0 .
$$

It follows from the intermediate value theorem that there exists a minimal $s_{m+1} \in\left(0, s_{m}\right)$ such that $f_{m+1}\left(s_{m+1}\right)=0$. In view of $(\mathrm{A} .27)$,

$$
f_{\infty}\left(s_{\infty}\right)=2\left(\frac{M}{1-s_{\infty}} \eta+\mu\right)+\frac{2 s_{\infty} L}{1-s_{\infty}} \eta+2 s_{\infty}(\ell-1)=0,
$$

by the choice of $s_{\infty}$. Note also that by $(3.3)$ and $(3.6), s_{\infty}$ exists in $(0,1)$.

The sequence $\left\{s_{m}\right\}$ is nonincreasing, bounded below by zero, and as such it converges to its infimum $s^{\star}$ satisfying $s^{\star} \geq s_{\infty}$. Hence, we showed (A.29). That completes the induction for (A.23) and (A.24).

Finally, the sequence $\left\{t_{n}\right\}$ is increasing, bounded above by $t^{\star \star}$, and as such it converges to its least upper bound $t^{\star}$.

That completes the proof of Lemma 3.1.

Proof of Lemma 4.7. If $L=0$, then (4.25) holds trivially. In this case, for $K>0$, an induction argument shows that

$$
t_{k+1}-t_{k}=\frac{2}{K}\left(2 h_{A}\right)^{2^{k}} \quad(k \geq 0),
$$

and therefore

$$
t_{k+1}=t_{1}+\left(t_{2}-t_{1}\right)+\cdots+\left(t_{k+1}-t_{k}\right)=\frac{2}{K} \sum_{m=0}^{k}\left(2 h_{A}\right)^{m},
$$

and

$$
t^{\star}=\lim _{k \rightarrow \infty} t_{k}=\frac{2}{K} \sum_{k=0}^{\infty}\left(2 h_{A}\right)^{2^{k}}
$$


Clearly, this series converges, since $k \leq 2^{k}, 2 h_{A}<1$, and is bounded above by the number

$$
\frac{2}{K} \sum_{k=0}^{\infty}\left(2 h_{A}\right)^{k}=s \frac{4}{K(2-K \eta)} .
$$

If $K=0$, then in view of (4.22) and $0 \leq L \leq K$, we deduce that $L=0$ and $t^{\star}=t_{k}=\eta(k \geq 1)$.

In the rest of the proof, we assume that $L>0$.

The result until estimate (4.26) follows from Lemma 2.1.

To show (4.27) we need the estimate

$$
\frac{1-(\delta / 2)^{k+1}}{1-\delta / 2} \eta \leq \frac{1}{L}\left(1-\left(\frac{\delta}{2}\right)^{k-1} \frac{K}{4 \bar{L}}\right) \quad(k \geq 1) .
$$

For $k=1$, (A.36) becomes

$$
\left(1+\frac{\delta}{2}\right) \eta \leq \frac{4 \bar{L}-K}{4 \bar{L} L}
$$

or

$$
\left(1+\frac{2 K}{K+\sqrt{K^{2}+8 L K}}\right) \eta \leq \frac{4 L-K+\sqrt{K^{2}+8 L K}}{L\left(4 L+K+\sqrt{K^{2}+8 L K}\right)} .
$$

In view of (4.20), it suffices to show

$$
\frac{L\left(4 L+K+\sqrt{K^{2}+8 L K}\right)\left(3 K+\sqrt{K^{2}+8 L K}\right)}{\left(K+\sqrt{K^{2}+8 L K}\right)\left(4 L-K+\sqrt{K^{2}+8 L K}\right)} \leq 2 \bar{L},
$$

which is true as equality.

Let us now assume estimate (A.36) is true for all integers smaller than or equal to $k$. We must show (A.36) holds for $k$ being $k+1$ :

$$
\frac{1-(\delta / 2)^{k+2}}{1-\delta / 2} \eta \leq \frac{1}{L}\left(1-\left(\frac{\delta}{2}\right)^{k} \frac{K}{4 \bar{L}}\right) \quad(k \geq 1) .
$$

or

$$
\left(1+\frac{\delta}{2}+\left(\frac{\delta}{2}\right)^{2}+\cdots+\left(\frac{\delta}{2}\right)^{k+1}\right) \eta \leq \frac{1}{L}\left(1-\left(\frac{\delta}{2}\right)^{k} \frac{K}{4 \bar{L}}\right) .
$$

By the induction hypothesis, to show (A.37), it suffices to prove

$$
\frac{1}{L}\left(1-\left(\frac{\delta}{2}\right)^{k-1} \frac{K}{4 \bar{L}}\right)+\left(\frac{\delta}{2}\right)^{k+1} \eta \leq \frac{1}{L}\left(1-\left(\frac{\delta}{2}\right)^{k} \frac{K}{4 \bar{L}}\right),
$$

or

$$
\left(\frac{\delta}{2}\right)^{k+1} \eta \leq \frac{1}{L}\left(\left(\frac{\delta}{2}\right)^{k-1}-\left(\frac{\delta}{2}\right)^{k}\right) \frac{K}{4 \bar{L}}
$$

or

$$
\delta^{2} \eta \leq \frac{K(2-\delta)}{2 \bar{L} L} .
$$


In view of (4.20) it suffices to show

$$
\frac{2 \bar{L} L \delta^{2}}{K(2-\delta)} \leq 2 \bar{L}
$$

which holds as equality by the choice of $\delta$ given by (4.24). That completes the induction for estimates (A.36).

We shall show (4.27) by induction on $k \geq 0$. First, (4.27) is true for $k=0$ by (4.20), (4.22), and (4.24). To show (4.27) for $k=1$, since $t_{2}-t_{1}=$ $\frac{K\left(t_{1}-t_{0}\right)^{2}}{2\left(1-L t_{1}\right)}$, it suffices to prove

$$
\frac{K \eta^{2}}{2(1-L \eta)} \leq \delta \bar{L} \eta^{2}
$$

or

$$
\frac{K}{1-L \eta} \leq \frac{8 \bar{L} K}{K+\sqrt{K^{2}+8 L K}} \quad(\eta \neq 0)
$$

or

$$
\eta \leq \frac{1}{L}\left(1-\frac{K+\sqrt{K^{2}+8 L K}}{8 \bar{L}}\right) \quad(L \neq 0, K \neq 0) .
$$

But by (4.20),

$$
\eta \leq \frac{4}{K+4 L+\sqrt{K^{2}+8 L K}}
$$

It then suffices to show

$$
\frac{4}{K+4 L+\sqrt{K^{2}+8 L K}} \leq \frac{1}{L}\left(1-\frac{K+\sqrt{K^{2}+8 L K}}{8 \bar{L}}\right),
$$

or

$$
\frac{K+\sqrt{K^{2}+8 L K}}{8 \bar{L}} \leq 1-\frac{4 L}{K+4 L+\sqrt{K^{2}+8 L K}},
$$

or

$$
\frac{K+\sqrt{K^{2}+8 L K}}{8 \bar{L}} \leq \frac{K+\sqrt{K^{2}+8 L K}}{K+4 L+\sqrt{K^{2}+8 L K}}
$$

which is true by (4.21).

Assume (A.37) holds for all integers smaller than or equal to $k$. We shall show it holds for $k$ replaced by $k+1$. 
Using (4.22) and the induction hypothesis, we have in turn

$$
\begin{aligned}
t_{k+2}-t_{k+1} & =\frac{K}{2\left(1-L t_{k+1}\right)}\left(t_{k+1}-t_{k}\right)^{2} \\
& \leq \frac{K}{2\left(1-L t_{k+1}\right)}\left(\left(\frac{\delta}{2}\right)^{k}\left(2 h_{A}\right)^{2^{k}-1} \eta\right)^{2} \\
& \leq \frac{K}{2\left(1-L t_{k+1}\right)}\left(\left(\frac{\delta}{2}\right)^{k-1}\left(2 h_{A}\right)^{-1} \eta\right)\left(\left(\frac{\delta}{2}\right)^{k+1}\left(2 h_{A}\right)^{2^{k+1}-1} \eta\right) \\
& \leq\left(\frac{\delta}{2}\right)^{k+1}\left(2 h_{A}\right)^{2^{k+1}-1} \eta
\end{aligned}
$$

since

$$
\frac{K}{2\left(1-L t_{k+1}\right)}\left(\left(\frac{\delta}{2}\right)^{k-1}\left(2 h_{A}\right)^{-1} \eta\right) \leq 1 \quad(k \geq 1) .
$$

Indeed, we can show, instead of (A.38),

$$
t_{k+1} \leq \frac{1}{L}\left(1-\left(\frac{\delta}{2}\right)^{k-1} \frac{K}{4 \bar{L}}\right)
$$

which is true, since by (4.26) and the induction hypothesis

$$
\begin{aligned}
t_{k+1} & \leq t_{k}+\frac{\delta}{2}\left(t_{k}-t_{k-1}\right) \\
& \leq t_{1}+\frac{\delta}{2}\left(t_{1}-t_{0}\right)+\cdots+\frac{\delta}{2}\left(t_{k}-t_{k-1}\right) \\
& \leq \eta+\left(\frac{\delta}{2}\right) \eta+\cdots+\left(\frac{\delta}{2}\right)^{k} \eta \\
& =\frac{1-(\delta / 2)^{k+1}}{1-\delta / 2} \eta \leq \frac{1}{L}\left(1-\left(\frac{\delta}{2}\right)^{k-1} \frac{K}{4 \bar{L}}\right) .
\end{aligned}
$$

That completes the induction for estimate (4.27).

Using estimate (A.37) for $j \geq k$, we obtain in turn, for $2 h_{A}<1$,

$$
\begin{aligned}
& \leq\left(\left(\frac{\delta}{2}\right)^{j}\left(2 h_{A}\right)^{2^{j}-1}+\left(\frac{\delta}{2}\right)^{j-1}\left(2 h_{A}\right)^{2^{j-1}-1}+\cdots+\left(\frac{\delta}{2}\right)^{k}\left(2 h_{A}\right)^{2^{k}-1}\right) \eta \\
& \leq\left(1+\left(2 h_{A}\right)^{2^{k}}+\left(\left(2 h_{A}\right)^{2^{k}}\right)^{2}+\cdots\right)\left(\frac{\delta}{2}\right)^{k}\left(2 h_{A}\right)^{2^{k}-1} \eta \\
& =\left(\frac{\delta}{2}\right)^{k} \frac{\left(2 h_{A}\right)^{2^{k}-1} \eta}{1-\left(2 h_{A}\right)^{2^{k}}}
\end{aligned}
$$

Estimate (4.28) follows from (A.39) by letting $j \rightarrow \infty$.

That completes the proof of Lemma 4.7. 
Conclusion. We provided a semilocal convergence analysis for (TSNTM) in order to approximate a locally unique solution of an equation in a Banach space.

Using our new idea of recurrent functions, a combination of Lipschitz and center-Lipschitz conditions, instead of only Lipschitz conditions, we provided an analysis with the following advantages over the works in [7]-[22]: weaker sufficient convergence conditions, tighter error bounds and larger convergence domain in some interesting cases. The efficiency of these methods was also discussed. Numerical examples and applications further validating the results were provided.

\section{References}

[1] I. K. Argyros, The Theory and Application of Abstract Polynomial Equations, CRC Press, Boca Raton, FL, 1998.

[2] - On the Newton-Kantorovich hypothesis for solving equations, J. Comput. Appl. Math. 169 (2004), 315-332.

[3] -, A unifying local-semilocal convergence analysis and applications for two-point Newton-like methods in Banach space, J. Math. Anal. Appl. 298 (2004), 374-397.

[4] -, Approximating solutions of equations using Newton's method with a modified Newton's method iterate as a starting point, Rev. Anal. Numér. Théor. Approx. 36 (2007), 123-137.

[5] - Approximating solutions of equations by combining Newton-like methods, J. Korea Soc. Educ. Ser. B Pure Appl. Math. 15 (2008), 35-45.

[6] -, Computational Theory of Iterative Methods, Stud. Comput. Math. 15, Elsevier, New York, 2007.

[7] - On a class of Newton-like methods for solving nonlinear equations, J. Comput. Appl. Math. 228 (2009), 115-122.

[8] S. Chandrasekhar, Radiative Transfer, Dover Publ., New York, 1960.

[9] X. Chen and T. Yamamoto, Convergence domains of certain iterative methods for solving nonlinear equations, Numer. Funct. Anal. Optim. 10 (1989), 37-48.

[10] J. E. Dennis, Toward a unified convergence theory for Newton-like methods, in: Nonlinear Functional Analysis and Applications, L. B. Rall (ed.), Academic Press, New York, 1971, 425-472.

[11] P. Deuflhard, Newton Methods for Nonlinear Problems. Affine Invariance and Adaptive Algorithms, Springer Ser. Comput. Math. 35, Springer, Berlin, 2004.

[12] P. Deuflhard and G. Heindl, Affine invariant convergence theorems for Newton's method and extensions to related methods, SIAM J. Numer. Anal. 16 (1979), 1-10.

[13] J. A. Ezquerro and M. A. Hernández, An optimization of Chebyshev's method, J. Complexity 25 (2009), 343-361.

[14] Z. Huang, A note on the Kantorovich theorem for Newton iteration, J. Comput. Appl. Math. 47 (1993), 211-217.

[15] L. V. Kantorovich and G. P. Akilov, Functional Analysis, Pergamon Press, Oxford, 1982.

[16] G. J. Miel, Majorizing sequences and error bounds for iterative methods, Math. Comp. 34 (1980), 185-202.

[17] I. Moret, A note on Newton type iterative methods, Computing 33 (1984), 65-73. 
[18] F. A. Potra, Sharp error bounds for a class of Newton-like methods, Libertas Math. 5 (1985), 71-84.

[19] W. C. Rheinboldt, A unified convergence theory for a class of iterative processes, SIAM J. Numer. Anal. 5 (1968), 42-63.

[20] T. Yamamoto, A convergence theorem for Newton-like methods in Banach spaces, Numer. Math. 51 (1987), 545-557.

[21] P. P. Zabrejko and D. F. Nguen, The majorant method in the theory of NewtonKantorovich approximations and the Pták error estimates, Numer. Funct. Anal. Optim. 9 (1987), 671-684.

[22] A. I. Zinčenko, Some approximate methods of solving equations with non-differentiable operators, Dopovidi Akad. Nauk Ukraïn. RSR 1963, 156-161 (in Ukrainian).

Department of Mathematics

Cameron University

Lawton, OK 73505, U.S.A.

E-mail: iargyros@cameron.edu
Laboratoire de Mathématiques et Applications Université de Poitiers Bd. Pierre et Marie Curie, Téléport 2, B.P. 30179 86962 Futuroscope Chasseneuil Cedex, France E-mail: said.hilout@math.univ-poitiers.fr

Received on 27.4.2008;

revised version on 7.7.2009 
